\title{
Thermodynamics of continuous media with intrinsic rotation and magnetoelectric coupling
}

Received: 11 August 2012 / Accepted: 16 February 2013

(C) Springer-Verlag Berlin Heidelberg 2013

\begin{abstract}
The thermodynamics of an electrically charged, multicomponent continuous medium with intrinsic rotation is analysed in the presence of electromagnetic fields with a weak linear magnetoelectric coupling in the non-relativistic limit. Taking into account the chemical composition of the current densities and stress tensors yields scalar dissipation terms accounting for chemical reactivities and vectorial dissipation terms accounting for transport. Three equations characterising the continuous medium are derived: a thermostatic equilibrium equation, a reversible and an irreversible thermodynamic evolution equation. Explicit expressions for the temperature and the chemical potentials are derived in terms of the electromagnetic fields and the magnetoelectric coupling. The transport equations contain electromagnetic terms normally not included in a standard thermodynamic phenomenology.
\end{abstract}

Keywords Thermodynamics · Continuous medium

\section{Introduction}

The aim of this publication is to establish a local thermodynamical description of continuous media with intrinsic rotation in the presence of electromagnetic fields with a weak linear magnetoelectric coupling. In many experimental situations, the system can be treated as a classical continuum on the scale of interest where the microscopic structure is smoothed out. In such as case, the local system is sufficiently large from a microscopic perspective to be treated classically, but it is sufficiently small from a macroscopic perspective to be considered as infinitesimal. In the present work, we follow essentially the approach of Stückelberg [1] and extend his formalism to account for intrinsic rotations and the presence of electromagnetic fields with a weak linear magnetoelectric coupling. We do not include the spontaneous electric polarisation and magnetisation and limit our analysis to the electric polarisation and magnetisation induced by the electrodynamic fields.

In most experimental conditions, the drift velocities of the chemical substances with respect to the laboratory rest frame are clearly non-relativistic. Thus, we restrict our thermodynamic analysis to a linear electromagnetic theory in the non-relativistic limit, which corresponds to the electric limit defined by Lebellac et al. [2]. In order to be able to define intensive fields such as the temperature, the chemical potential, the electrostatic potential, the electric field and the magnetic induction field, we require the local infinitesimal system to be homogeneous, uniform and at equilibrium.

Communicated by Andreas Öchsner.

S. D. Brechet $(\bowtie)$ - J.-P. Ansermet

ICMP EPFL, 1015 Lausanne, Switzerland

E-mail: sylvain.brechet@epfl.ch

J.-P. Ansermet

E-mail: jean-philippe.ansermet@epfl.ch 
We introduce phenomenological relations that express, in terms of the chemical constituents of the continuous medium, the mass density, the electric charge density, the intrinsic angular mass density, the momentum and the intrinsic angular momentum. Using these phenomenological relations, we express the stress and angular stress tensors in terms of the chemical current densities. For a local thermodynamic equilibrium, we derive explicit expressions for the temperature and the chemical potentials in terms of the electromagnetic fields and the magnetoelectric coupling. Furthermore, we derive the linear phenomenological relations from the irreversible evolution.

The structure of this publication is as follows. In Sect. 2, we first define the system by choosing the appropriate state fields. Then, we use a phenomenological approach to obtain the linear constitutive electrodynamic relations in the presence of a weak linear magnetoelectric coupling. We also establish the continuity equations of the relevant physical fields. In Sect. 3, we determine the time evolution equations of the system in terms of the chemical composition, and we obtain explicit expressions for the temperature and the chemical potentials in terms of the electromagnetic fields in the presence of a weak magnetoelectric coupling. Finally, in Sect. 4, we establish the linear phenomenological relations and discuss physical applications.

\section{Thermodynamic description}

\subsection{Thermodynamic state fields}

The state of a continuous medium is defined by a set of state variables. The local state of a continuous medium is defined by a set of state fields that are function of the space and time coordinates. To keep the notation concise, we do not explicitly write the space and time dependence of the state fields. The local thermodynamic state of a continuous medium consisting of $N$ chemical substances with intrinsic rotation in the presence of electromagnetic fields is determined by the following state fields:

- the entropy density field $s$,

- the number density fields $n_{A}$ of $N$ chemical substances where $A \in\{1, \ldots, N\}$,

- the velocity field $\mathbf{v}$,

- the intrinsic angular velocity field $\omega$,

- the electric displacement field $\mathbf{D}$,

- the magnetic induction field $\mathbf{B}$.

Note that the electromagnetic fields $\mathbf{D}$ and $\mathbf{B}$ are frame independent in the non-relativistic limit, i.e., $\mathbf{v}^{2} / c^{2} \ll 1$, where $c$ represents the speed of light in the vacuum [3].

The local material frame is defined as the Lagrangian frame where the centre of mass of the local element of continuous medium is at rest (i.e. $\mathbf{v}=\mathbf{0}$ ). It should not be confused with the local rest frame, which is characterised by the absence of translational and intrinsic rotational motion (i.e. $\mathbf{v}=\mathbf{0}$ and $\boldsymbol{\omega}=\mathbf{0}$ ).

\subsection{Linear electromagnetic constitutive relations}

In the local material frame, the electromagnetic constitutive relations relate the electromagnetic fields representing densities of extensive electromagnetic properties to the electromagnetic fields corresponding to intensive electromagnetic properties. The extensive electromagnetic properties are the electric dipoles and the magnetic moments of the physical system. The corresponding densities are the electric polarisation and the magnetisation.

In the presence of a magnetoelectric coupling in a linear electromagnetic framework, the total electric polarisation is given by the electric displacement field $\mathbf{D}\left(s, n_{A}, \mathbf{E}, \mathbf{B}\right)$, that is a function of the entropy density $s$ and the densities $n_{A}$ of chemical substances $A$, and a linear mapping of the electric field $\mathbf{E}$ and the magnetic induction field $\mathbf{B}$. Note that the electromagnetic fields $\mathbf{E}$ and $\mathbf{B}$ are intensive fields. The total electric polarisation is split into the electric polarisation of the vacuum and the electric polarisation of the matter, i.e.,

$$
\mathbf{D}\left(s, n_{A}, \mathbf{E}, \mathbf{B}\right)=\varepsilon_{0} \mathbf{E}+\mathbf{P}\left(s, n_{A}, \mathbf{E}, \mathbf{B}\right),
$$

where $\varepsilon_{0}$ is the vacuum permittivity, and $\mathbf{P}\left(s, n_{A}, \mathbf{E}, \mathbf{B}\right)$ is the electric polarisation of the matter. In the absence of a spontaneous electric polarisation, the electric polarisation of the matter $\mathbf{P}\left(s, n_{A}, \mathbf{E}, \mathbf{B}\right)$ is linearly induced by the electric field $\mathbf{E}$ and the magnetic induction field $\mathbf{B}$, i.e., 


$$
\mathbf{P}\left(s, n_{A}, \mathbf{E}, \mathbf{B}\right)=\varepsilon_{0} \chi_{e}\left(s, n_{A}\right) \cdot \mathbf{E}+c \varepsilon_{0} \chi_{\alpha}\left(s, n_{A}\right) \cdot \mathbf{B},
$$

where $c$ is the speed of light in the vacuum, $\chi_{e}\left(s, n_{A}\right)$ is the electric susceptibility tensor, and $\chi_{\alpha}\left(s, n_{A}\right)$ is the magnetoelectric susceptibility tensor. Note that these tensors are dimensionless.

In the presence of a magnetoelectric coupling in a linear electromagnetic framework, the total magnetisation is given by the opposite of the magnetic field $\mathbf{H}\left(s, n_{A}, \mathbf{E}, \mathbf{B}\right)$, that is a function of the entropy density $s$ and the densities $n_{A}$ of chemical substances $A$, and a linear mapping of the electric field $\mathbf{E}$ and the magnetic induction field $\mathbf{B}$. It is split into the magnetisation of the vacuum and the magnetisation of the matter, i.e.,

$$
-\mathbf{H}\left(s, n_{A}, \mathbf{E}, \mathbf{B}\right)=-\mu_{0}^{-1} \mathbf{B}+\mathbf{M}\left(s, n_{A}, \mathbf{E}, \mathbf{B}\right),
$$

where $\mathbf{M}\left(s, n_{A}, \mathbf{E}, \mathbf{B}\right)$ is the magnetisation of the matter, and $\mu_{0}$ is the vacuum, which satisfies $c^{-2}=\varepsilon_{0} \mu_{0}$. Note that the negative signs in the relation (3) are a consequence of the historical choice of definition for $\mathbf{M}$. In the absence of a spontaneous magnetisation, the magnetisation of the matter $\mathbf{M}\left(s, n_{A}, \mathbf{E}, \mathbf{B}\right)$ is linearly induced by the electric field $\mathbf{E}$ and the magnetic induction field $\mathbf{B}$, i.e.,

$$
\mathbf{M}\left(s, n_{A}, \mathbf{E}, \mathbf{B}\right)=c^{-1} \mu_{0}^{-1} \chi_{\alpha}\left(s, n_{A}\right) \cdot \mathbf{E}+\mu_{0}^{-1} \chi_{m}\left(s, n_{A}\right) \cdot \mathbf{B},
$$

where $\chi_{m}\left(s, n_{A}\right)$ is the magnetic susceptibility tensor. Note that the same magnetoelectric susceptibility tensor $\chi_{\alpha}\left(s, n_{A}\right)$ appears in the expressions (2) and (4) for the electric polarisation and the magnetisation of the matter, respectively. This is due to the fact that $\chi_{\alpha}\left(s, n_{A}\right)$ represents geometric properties of the matter.

The electric permittivity tensor $\boldsymbol{\varepsilon}\left(s, n_{A}\right)$, the magnetic permeability tensor $\boldsymbol{\mu}\left(s, n_{A}\right)$ and the magnetoelectric coupling tensor $\boldsymbol{\alpha}\left(s, n_{A}\right)$ are, respectively, defined as,

$$
\begin{aligned}
& \boldsymbol{\varepsilon}\left(s, n_{A}\right)=\varepsilon_{0}\left(\mathbb{1}+\chi_{e}\left(s, n_{A}\right)\right) \\
& \boldsymbol{\mu}\left(s, n_{A}\right)=\mu_{0}\left(\mathbb{1}-\chi_{m}\left(s, n_{A}\right)\right)^{-1}=c^{-2} \varepsilon_{0}^{-1}\left(\mathbb{1}-\chi_{m}\left(s, n_{A}\right)\right)^{-1}, \\
& \boldsymbol{\alpha}\left(s, n_{A}\right)=c \varepsilon_{0} \chi_{\alpha}\left(s, n_{A}\right)=c^{-1} \mu_{0}^{-1} \chi_{\alpha}\left(s, n_{A}\right),
\end{aligned}
$$

where $\mathbb{1}$ is the identity rank-2 tensor. Using the definitions (5), the electric constitutive relation (1), and the magnetic constitutive relation (3) are recast, respectively, as,

$$
\begin{aligned}
& \mathbf{D}\left(s, n_{A}, \mathbf{E}, \mathbf{B}\right)=\boldsymbol{\varepsilon}\left(s, n_{A}\right) \cdot \mathbf{E}+\boldsymbol{\alpha}\left(s, n_{A}\right) \cdot \mathbf{B}, \\
& \mathbf{H}\left(s, n_{A}, \mathbf{E}, \mathbf{B}\right)=\boldsymbol{\alpha}\left(s, n_{A}\right) \cdot \mathbf{E}+\boldsymbol{\mu}^{-1}\left(s, n_{A}\right) \cdot \mathbf{B} .
\end{aligned}
$$

Since the electric fields $\mathbf{D}$ and $\mathbf{E}$ are vectors and the magnetic fields $\mathbf{H}$ and $\mathbf{B}$ are pseudovectors, the invariance of the constitutive relations (9) under inversion implies that the phenomenological tensors $\boldsymbol{\varepsilon}\left(s, n_{A}\right)$ and $\boldsymbol{\mu}\left(s, n_{A}\right)$ are symmetric and the phenomenological tensor $\boldsymbol{\alpha}\left(s, n_{A}\right)$ is antisymmetric, i.e.,

$$
\begin{aligned}
& \boldsymbol{\varepsilon}^{T}\left(s, n_{A}\right)=\boldsymbol{\varepsilon}\left(s, n_{A}\right), \\
& \boldsymbol{\mu}^{T}\left(s, n_{A}\right)=\boldsymbol{\mu}\left(s, n_{A}\right), \\
& \boldsymbol{\alpha}^{T}\left(s, n_{A}\right)=-\boldsymbol{\alpha}\left(s, n_{A}\right) .
\end{aligned}
$$

where the exponent ${ }^{T}$ denotes the transpose. Since the tensor $\boldsymbol{\alpha}\left(s, n_{A}\right)$ is antisymmetric, the electric polarisation (2) induced by the magnetic induction field $\mathbf{B}$ and the magnetisation (4) induced by the electric field $\mathbf{E}$ can be recast in terms of the dual vector $\hat{\boldsymbol{\alpha}}\left(s, n_{A}\right)$ of $\boldsymbol{\alpha}\left(s, n_{A}\right)$, i.e.,

$$
\begin{aligned}
& \mathbf{P}\left(s, n_{A}, \mathbf{0}, \mathbf{B}\right)=\boldsymbol{\alpha}\left(s, n_{A}\right) \cdot \mathbf{B}=\hat{\boldsymbol{\alpha}}\left(s, n_{A}\right) \times \mathbf{B}, \\
& \mathbf{M}\left(s, n_{A}, \mathbf{E}, \mathbf{0}\right)=\boldsymbol{\alpha}\left(s, n_{A}\right) \cdot \mathbf{E}=\hat{\boldsymbol{\alpha}}\left(s, n_{A}\right) \times \mathbf{E} .
\end{aligned}
$$

This implies that $\mathbf{P}\left(s, n_{A}, \mathbf{0}, \mathbf{B}\right)$ is orthogonal to $\mathbf{B}$ and $\mathbf{M}\left(s, n_{A}, \mathbf{E}, \mathbf{0}\right)$ is orthogonal to $\mathbf{E}$.

Experimentally, magnetoelectric effects are known to be much smaller than electromagnetic effects. The weakness of the magnetoelectric coupling is expressed in terms of the phenomenological tensors as,

$$
\boldsymbol{\alpha}^{2}\left(s, n_{A}\right) \ll \boldsymbol{\varepsilon}^{\mathbf{- 1}}\left(s, n_{A}\right) \cdot \boldsymbol{\mu}\left(s, n_{A}\right) .
$$


Under the weak coupling condition (8), the inverse of the electromagnetic constitutive relations (6) is expressed in terms of the fields $s, n_{A}, \mathbf{D}$ and $\mathbf{H}$ as,

$$
\begin{aligned}
& \mathbf{E}\left(s, n_{A}, \mathbf{D}, \mathbf{H}\right)=\boldsymbol{\varepsilon}^{-1}\left(s, n_{A}\right) \cdot \mathbf{D}-\left(\boldsymbol{\varepsilon}^{-1}\left(s, n_{A}\right) \cdot \boldsymbol{\alpha}\left(s, n_{A}\right) \cdot \boldsymbol{\mu}\left(s, n_{A}\right)\right) \cdot \mathbf{H}, \\
& \mathbf{B}\left(s, n_{A}, \mathbf{D}, \mathbf{H}\right)=-\left(\boldsymbol{\mu}\left(s, n_{A}\right) \cdot \boldsymbol{\alpha}\left(s, n_{A}\right) \cdot \boldsymbol{\varepsilon}^{-1}\left(s, n_{A}\right)\right) \cdot \mathbf{D}+\boldsymbol{\mu}\left(s, n_{A}\right) \cdot \mathbf{H} .
\end{aligned}
$$

Under the same condition, the electromagnetic constitutive relations (6) and (9) are recast in terms of the fields $s, n_{A}, \mathbf{D}$ and $\mathbf{B}$ as,

$$
\begin{aligned}
& \mathbf{E}\left(s, n_{A}, \mathbf{D}, \mathbf{B}\right)=\boldsymbol{\varepsilon}^{-1}\left(s, n_{A}\right) \cdot \mathbf{D}-\left(\boldsymbol{\varepsilon}^{-1}\left(s, n_{A}\right) \cdot \boldsymbol{\alpha}\left(s, n_{A}\right)\right) \cdot \mathbf{B}, \\
& \mathbf{H}\left(s, n_{A}, \mathbf{D}, \mathbf{B}\right)=\left(\boldsymbol{\alpha}\left(s, n_{A}\right) \cdot \boldsymbol{\varepsilon}^{-1}\left(s, n_{A}\right)\right) \cdot \mathbf{D}+\boldsymbol{\mu}^{-1}\left(s, n_{A}\right) \cdot \mathbf{B},
\end{aligned}
$$

and in terms of the fields $s, n_{A}, \mathbf{E}$ and $\mathbf{H}$ as,

$$
\begin{aligned}
& \mathbf{D}\left(s, n_{A}, \mathbf{E}, \mathbf{H}\right)=\boldsymbol{\varepsilon}\left(s, n_{A}\right) \cdot \mathbf{E}+\left(\boldsymbol{\alpha}\left(s, n_{A}\right) \cdot \boldsymbol{\mu}\left(s, n_{A}\right)\right) \cdot \mathbf{H}, \\
& \mathbf{B}\left(s, n_{A}, \mathbf{E}, \mathbf{H}\right)=-\left(\boldsymbol{\mu}\left(s, n_{A}\right) \cdot \boldsymbol{\alpha}\left(s, n_{A}\right)\right) \cdot \mathbf{E}+\boldsymbol{\mu}\left(s, n_{A}\right) \cdot \mathbf{H} .
\end{aligned}
$$

\subsection{Matter continuity equations}

The time evolution of every extensive physical property in a local infinitesimal system is expressed by a continuity equation, which is a local detailed balance equation. In this publication, we shall refer to the continuity equations expressed in terms of the material time derivative as the material continuity equations.

The material continuity equation for an extensive scalar property $F$ is given by,

$$
\dot{f}+(\nabla \cdot \mathbf{v}) f+\nabla \cdot \mathbf{j}_{f}=\rho_{f},
$$

where $\dot{f}$ is the material time derivative of the scalar density $f, \mathbf{j}_{f}$ is the vectorial diffusive current density, and $\rho_{f}$ is the scalar source density of $F$. Note that the frame-independent material time derivative operator is related to the partial time derivative operator by,

$$
\cdot=\partial_{t}+(\mathbf{v} \cdot \nabla)
$$

The material continuity equation for an extensive vectorial property $\mathbf{F}$ is given by,

$$
\dot{\mathbf{f}}+(\nabla \cdot \mathbf{v}) \mathbf{f}+\nabla \cdot \mathrm{j}_{\mathbf{f}}=\rho_{\mathbf{f}},
$$

where $\dot{\mathbf{f}}$ is the material time derivative of the vectorial density $\mathbf{f}, j_{\mathbf{f}}$ is the rank-2 tensorial diffusive current density, and $\boldsymbol{\rho}_{\mathrm{f}}$ is the vectorial source density of $\mathbf{F}$.

The material continuity Eq. (12) for the entropy yields,

$$
\dot{s}+(\nabla \cdot \mathbf{v}) s+\nabla \cdot \mathbf{j}_{s}=\rho_{s} \geqslant 0,
$$

where $s$ is the entropy density, $\mathbf{j}_{s}$ is the diffusive entropy current density, and $\rho_{s}$ is the entropy source density that is positive definite in order to satisfy locally the second law of thermodynamics.

The material continuity Eq. (12) for the number of elementary units of chemical substance $A$ is given by,

$$
\dot{n}_{A}+(\nabla \cdot \mathbf{v}) n_{A}+\nabla \cdot \mathbf{j}_{A}=\sum_{a} \omega_{a} v_{a A}
$$

where $n_{A}$ is the number density of the chemical substance $A, \mathbf{j}_{A}$ is the diffusive current density, and the source density $\rho_{A}$ is expressed explicitly as the sum over all the chemical reactions $a$ of the product of the stoichiometric coefficients $v_{a A}$ and the reaction rate densities $\omega_{a}$. 


\subsection{Electromagnetic continuity equations}

Electromagnetic phenomena are locally described by the Maxwell equations. These equations consist of two spatial constraint equations and two continuity equations. The constraint equations are Gauss' law and Thomson's law, respectively, given by,

$$
\begin{aligned}
& \nabla \cdot \mathbf{D}=q, \\
& \nabla \cdot \mathbf{B}=0,
\end{aligned}
$$

where $q$ is the electric charge density. The continuity equations are Ampère-Maxwell's law and Faraday's law, which are expressed in an Eulerian frame where the matter has a velocity $\mathbf{v}$ as,

$$
\begin{aligned}
& \partial_{t} \mathbf{D}+\mathbf{j}=\nabla \times \mathbf{H}^{\prime}, \\
& \partial_{t} \mathbf{B}=-\nabla \times \mathbf{E}^{\prime},
\end{aligned}
$$

where $\mathbf{E}^{\prime}$ and $\mathbf{H}^{\prime}$ are, respectively, the electric and magnetic fields in that frame. Note that the electric displacement field $\mathbf{D}$ and the magnetic induction field $\mathbf{B}$ are the dynamic fields in Maxwell's equation (18) and Faraday's equation (19), respectively, whereas the electric field $\mathbf{E}^{\prime}$ and the magnetic field $\mathbf{H}^{\prime}$ are auxiliary fields. Therefore, we choose $\mathbf{D}$ and $\mathbf{B}$ as state fields.

In the non-relativistic limit [2] in the local material frame, the transformation laws relating the electric field $\mathbf{E}^{\prime}$ and magnetic field $\mathbf{H}^{\prime}$ in the Eulerian frame where the matter has a velocity $\mathbf{v}$ to the electric field $\mathbf{E}$ and magnetic field $\mathbf{H}$ in the local material frame where the matter is at rest, i.e., $\mathbf{v}=\mathbf{0}$, are given by [3],

$$
\begin{aligned}
& \mathbf{E}^{\prime}\left(s, n_{A}, \mathbf{v}, \mathbf{D}, \mathbf{B}\right)=\mathbf{E}\left(s, n_{A}, \mathbf{D}, \mathbf{B}\right)-\mathbf{v} \times \mathbf{B}, \\
& \mathbf{H}^{\prime}\left(s, n_{A}, \mathbf{v}, \mathbf{D}, \mathbf{B}\right)=\mathbf{H}\left(s, n_{A}, \mathbf{D}, \mathbf{B}\right)+\mathbf{v} \times \mathbf{D} .
\end{aligned}
$$

Using Gauss' law (16), Thomson's law (17), the transformation laws (20) and the definition of the time differential operator in the local material frame, i.e.,

$$
\cdot \partial_{t}+\mathbf{v} \cdot \nabla
$$

Ampère-Maxwell's law (18) and Faraday's law (19) are, respectively, recast in the local material frame as,

$$
\begin{aligned}
& \dot{\mathbf{D}}+(\nabla \cdot \mathbf{v}) \mathbf{D}-(\mathbf{D} \cdot \nabla) \mathbf{v}+\mathbf{j}_{q}=\nabla \times \mathbf{H}, \\
& \dot{\mathbf{B}}+(\nabla \cdot \mathbf{v}) \mathbf{B}-(\mathbf{B} \cdot \nabla) \mathbf{v}=-\nabla \times \mathbf{E},
\end{aligned}
$$

where $\mathbf{j}_{q}$ is the diffusive electric current density defined as the electric current density in the local material frame, which is related to the electric current density by,

$$
\mathbf{j}=q \mathbf{v}+\mathbf{j}_{q}
$$

Gauss' law (16), the definition (21) and the relation (24) imply that the divergence of Ampère-Maxwell's law (18) yields the continuity equation for the electric charge, i.e.,

$$
\dot{q}+(\nabla \cdot \mathbf{v}) q+\nabla \cdot \mathbf{j}_{q}=0 .
$$

\subsection{Energy continuity equation}

The continuity equation for the energy is given by,

$$
\dot{e}+(\nabla \cdot \mathbf{v}) e+\nabla \cdot \mathbf{j}_{e}=\mathbf{f}^{\mathrm{ext}} \cdot \mathbf{v}+\boldsymbol{\tau}^{\mathrm{ext}} \cdot \boldsymbol{\omega},
$$

where $e\left(s, n_{A}, \mathbf{v}, \boldsymbol{\omega}, \mathbf{D}, \mathbf{B}\right)$ is the energy density, $\mathbf{j}_{e}$ is the energy current density, $\mathbf{f}^{\text {ext }}$, and $\boldsymbol{\tau}^{\text {ext }}$ are, respectively, the external force and torque densities of the non-electromagnetic fields. Note that in our thermodynamical approach, the electromagnetic force and torque densities are exerted by the electromagnetic fields on the matter fields. Since the matter fields and the electromagnetic fields are part of the local system, the electromagnetic force and torque densities are considered, respectively, as "internal" force and torque densities of the local system. 
The energy density $e\left(s, n_{A}, \mathbf{v}, \omega, \mathbf{D}, \mathbf{B}\right)$ can be split into a kinetic and an internal part according to,

$$
e\left(s, n_{A}, \mathbf{v}, \boldsymbol{\omega}, \mathbf{D}, \mathbf{B}\right)=\frac{1}{2} m\left(s, n_{A}\right) \mathbf{v}^{2}+\frac{1}{2} I\left(s, n_{A}\right) \omega^{2}+u\left(s, n_{A}, \mathbf{D}, \mathbf{B}\right),
$$

where $m\left(s, n_{A}\right)$ is the mass density, $I\left(s, n_{A}\right)$ is the intrinsic angular mass moment with respect to the intrinsic angular velocity $\omega$, and $u\left(s, n_{A}, \mathbf{D}, \mathbf{B}\right)=e\left(s, n_{A}, \mathbf{0}, \mathbf{0}, \mathbf{D}, \mathbf{B}\right)$ is the internal energy density, which is defined as the energy density in the local rest frame of the continuous medium where $\mathbf{v}=\mathbf{0}$ and $\boldsymbol{\omega}=\mathbf{0}$.

The analytical expression for the internal energy density $u\left(s, n_{A}, \mathbf{D}, \mathbf{B}\right)$ can be split as,

$$
u\left(s, n_{A}, \mathbf{D}, \mathbf{B}\right)=u^{\mathrm{mat}}\left(s, n_{A}\right)+u^{\mathrm{em}}\left(s, n_{A}, \mathbf{D}, \mathbf{B}\right),
$$

where $u^{\text {mat }}\left(s, n_{A}\right) \equiv u\left(s, n_{A}, \mathbf{0}, \mathbf{0}\right)$ is the internal energy density of the matter fields, and $u^{\mathrm{em}}\left(s, n_{A}, \mathbf{D}, \mathbf{B}\right)$ is the internal energy density of the electromagnetic fields and their interaction with the matter fields.

The electromagnetic fields $\mathbf{E}$ and $\mathbf{H}$ are defined in the local material frame as the conjugate fields of the electromagnetic state fields $\mathbf{D}$ and $\mathbf{B}$, respectively, i.e.,

$$
\begin{aligned}
& \mathbf{E}\left(s, n_{A}, \mathbf{D}, \mathbf{B}\right) \equiv \frac{\partial u\left(s, n_{A}, \mathbf{D}, \mathbf{B}\right)}{\partial \mathbf{D}}, \\
& \mathbf{H}\left(s, n_{A}, \mathbf{D}, \mathbf{B}\right) \equiv \frac{\partial u\left(s, n_{A}, \mathbf{D}, \mathbf{B}\right)}{\partial \mathbf{B}} .
\end{aligned}
$$

The analytical expression for the electromagnetic part of the internal energy density $u^{\mathrm{em}}\left(s, n_{A}, \mathbf{D}, \mathbf{B}\right)$ has to satisfy the integrability conditions (29) and the symmetries of the phenomenological tensors that appear in the constitutive equations (10), i.e.,

$$
\begin{aligned}
u^{\mathrm{em}}\left(s, n_{A}, \mathbf{D}, \mathbf{B}\right)= & \frac{1}{2}\left(\boldsymbol{\varepsilon}^{-1}\left(s, n_{A}\right) \cdot(\mathbf{D} \odot \mathbf{D})+\boldsymbol{\mu}^{-1}\left(s, n_{A}\right) \cdot(\mathbf{B} \odot \mathbf{B})\right) \\
& -\left(\varepsilon^{-1}\left(s, n_{A}\right) \cdot \boldsymbol{\alpha}\left(s, n_{A}\right)\right) \cdot(\mathbf{D} \otimes \mathbf{B})
\end{aligned}
$$

where the symbols $\odot$ and $\otimes$ denote, respectively, a symmetrised and an antisymmetrised tensorial product. Using the constitutive relations (10), the electromagnetic part of the internal energy density (30) is recast as,

$$
u^{\mathrm{em}}\left(s, n_{A}, \mathbf{D}, \mathbf{B}\right)=\frac{1}{2}\left(\mathbf{E}\left(s, n_{A}, \mathbf{D}, \mathbf{B}\right) \cdot \mathbf{D}+\mathbf{H}\left(s, n_{A}, \mathbf{D}, \mathbf{B}\right) \cdot \mathbf{B}\right) .
$$

The electromagnetic part of the internal energy density (30) can be expressed as a quadratic form in a six dimensions, i.e.,

$$
u^{\mathrm{em}}\left(s, n_{A}, \mathbf{D}, \mathbf{B}\right)=\frac{1}{2} \varepsilon_{0}^{-1} \chi\left(s, n_{A}\right) \cdot\left(\left(\mathbf{D}, \varepsilon_{0} c \mathbf{B}\right) \odot\left(\mathbf{D}, \varepsilon_{0} c \mathbf{B}\right)\right),
$$

where $\left(\mathbf{D}, \varepsilon_{0} c \mathbf{B}\right)$ is a vector in six dimensions, and $\chi\left(s, n_{A}\right)$ is a rank-2 dimensionless tensor in six dimensions that is expressed in blocks as,

$$
\chi\left(s, n_{A}\right)=\left(\begin{array}{cc}
\varepsilon_{0} \boldsymbol{\varepsilon}^{-1}\left(s, n_{A}\right) & -c^{-1} \boldsymbol{\varepsilon}^{-1}\left(s, n_{A}\right) \cdot \boldsymbol{\alpha}\left(s, n_{A}\right) \\
c^{-1} \boldsymbol{\alpha}\left(s, n_{A}\right) \cdot \boldsymbol{\varepsilon}^{-1}\left(s, n_{A}\right) & \mu_{0} \boldsymbol{\mu}^{-1}\left(s, n_{A}\right)
\end{array}\right)
$$

Since the electromagnetic part of the internal energy density $u^{\mathrm{em}}\left(s, n_{A}, \mathbf{D}, \mathbf{B}\right)$ can be expressed as a positivedefinite quadratic form (32), it guarantees the existence of a minimum of $u^{\mathrm{em}}\left(s, n_{A}, \mathbf{D}, \mathbf{B}\right)$ under a variation of the dynamical electromagnetic fields $\mathbf{D}$ and $\mathbf{B}$. Thus, the expression (30) of the electromagnetic part of the internal energy density is the unique physically consistent solution in the presence of a magnetoelectric coupling in a linear electromagnetic framework. 
2.6 Momentum and mass continuity equations

The continuity equation for the momentum is of the form,

$$
\dot{\mathbf{p}}+(\nabla \cdot \mathbf{v}) \mathbf{p}-\nabla \cdot \sigma=\mathbf{f}^{\mathrm{ext}},
$$

where $\mathbf{p}\left(s, n_{A}, \mathbf{v}, \mathbf{D}, \mathbf{B}\right)$ is the momentum density, and $\boldsymbol{\sigma}\left(s, n_{A}, \mathbf{v}, \mathbf{D}, \mathbf{B}\right)$ is the stress density tensor. Note that the momentum density and the stress density tensor are not functions of the intrinsic angular velocity $\omega$, since such fields are independent of intrinsic rotations around the local centre of mass.

The momentum density consists of two parts: a matter part $\mathbf{p}^{\text {mat }}\left(s, n_{A}, \mathbf{v}\right)$ and an electromagnetic part $\mathbf{p}^{\mathrm{em}}(\mathbf{D}, \mathbf{B})$, i.e.,

$$
\mathbf{p}\left(s, n_{A}, \mathbf{v}, \mathbf{D}, \mathbf{B}\right)=\mathbf{p}^{\text {mat }}\left(s, n_{A}, \mathbf{v}\right)+\mathbf{p}^{\mathrm{em}}(\mathbf{D}, \mathbf{B})
$$

where

$$
\begin{aligned}
& \mathbf{p}^{\text {mat }}\left(s, n_{A}, \mathbf{v}\right)=m\left(s, n_{A}\right) \mathbf{v}, \\
& \mathbf{p}^{\mathrm{em}}(\mathbf{D}, \mathbf{B})=\mathbf{D} \times \mathbf{B} .
\end{aligned}
$$

Similarly, the stress density tensor also $\boldsymbol{\sigma}\left(s, n_{A}, \mathbf{v}, \mathbf{D}, \mathbf{B}\right)$ consists of two parts: a matter part $\boldsymbol{\sigma}^{\text {mat }}\left(s, n_{A}, \mathbf{v}\right)$ and an electromagnetic part $\boldsymbol{\sigma}^{\mathrm{em}}\left(s, n_{A}, \mathbf{D}, \mathbf{B}\right)$, i.e.,

$$
\boldsymbol{\sigma}\left(s, n_{A}, \mathbf{v}, \mathbf{D}, \mathbf{B}\right)=\boldsymbol{\sigma}^{\mathrm{mat}}\left(s, n_{A}, \mathbf{v}\right)+\boldsymbol{\sigma}^{\mathrm{em}}\left(s, n_{A}, \mathbf{D}, \mathbf{B}\right) .
$$

The relations (34) and (37) imply that the continuity equation (33) can be split according to

$$
\begin{aligned}
& \dot{\mathbf{p}}^{\mathrm{mat}}+(\nabla \cdot \mathbf{v}) \mathbf{p}^{\mathrm{mat}}-\nabla \cdot \boldsymbol{\sigma}^{\mathrm{mat}}=\mathbf{f}^{\mathrm{ext}}+\mathbf{f}^{\mathrm{em}}, \\
& \dot{\mathbf{p}}^{\mathrm{em}}+(\nabla \cdot \mathbf{v}) \mathbf{p}^{\mathrm{em}}-\nabla \cdot \sigma^{\mathrm{em}}=-\mathbf{f}^{\mathrm{em}},
\end{aligned}
$$

where $\mathbf{f}^{\mathrm{em}}$ is the electromagnetic force density exerted by the electromagnetic fields on the matter fields. Note that the electromagnetic force density $\mathbf{f}^{\mathrm{em}}$ is an internal force density for the whole system. However, it is considered as an "external" force density for the matter subsystem.

Using the expression (35) for the momentum density of the matter part, the continuity equation (38) for the momentum density of the matter part becomes,

$$
m \dot{\mathbf{v}}+(\dot{m}+(\nabla \cdot \mathbf{v}) m) \mathbf{v}=\mathbf{f}^{\mathrm{ext}}+\mathbf{f}^{\mathrm{em}}+\nabla \cdot \boldsymbol{\sigma}^{\mathrm{mat}},
$$

which is Newton's second law in translation. The translational symmetry of the Galilean group implies that this law is independent of the velocity $\mathbf{v}$. Thus, the second term on the LHS of the equation of motion (40) has to vanish, i.e.,

$$
\dot{m}+(\nabla \cdot \mathbf{v}) m=0,
$$

which is the continuity equation for the mass.

Using the continuity equation (41), Newton's second law of motion in translation reduces to,

$$
m \dot{\mathbf{v}}=\mathbf{f}^{\mathrm{ext}}+\mathbf{f}^{\mathrm{em}}+\nabla \cdot \boldsymbol{\sigma}^{\mathrm{mat}},
$$

and corresponds to one of the Navier-Stokes equations for an electrically charged continuous medium with electromagnetic fields. Note that $\nabla \cdot \sigma^{\text {mat }}$ is the mechanical shear force density.

The scalar product $\left(\dot{\mathbf{p}}^{\mathrm{em}}+(\boldsymbol{\nabla} \cdot \mathbf{v}) \mathbf{p}^{\mathrm{em}}\right) \cdot \mathbf{v}$ in the continuity equation (39) is a relativistic correction. Thus, in a non-relativistic framework, the scalar product of the continuity equation (39) and the velocity vector $\mathbf{v}$ reduces to,

$$
\left(\nabla \cdot \sigma^{\mathrm{em}}\right) \cdot \mathbf{v}=\mathbf{f}^{\mathrm{em}} \cdot \mathbf{v}
$$


Maxwell-Ampère's law (22) and Faraday's law (23) imply that the scalar product of the continuity equation (39) and the velocity field $\mathbf{v}$ can be expressed as,

$$
\begin{aligned}
& \left((\mathbf{D} \times \mathbf{B})^{\cdot}+2(\boldsymbol{\nabla} \cdot \mathbf{v})(\mathbf{D} \times \mathbf{B})-((\mathbf{D} \cdot \boldsymbol{\nabla}) \mathbf{v}) \times \mathbf{B}-\mathbf{D} \times((\mathbf{B} \cdot \boldsymbol{\nabla}) \mathbf{v})\right) \cdot \mathbf{v} \\
& \quad=\left(-\mathbf{j}_{q} \times \mathbf{B}-\mathbf{D} \times(\boldsymbol{\nabla} \times \mathbf{E})-\mathbf{B} \times(\boldsymbol{\nabla} \times \mathbf{H})\right) \cdot \mathbf{v},
\end{aligned}
$$

where the terms on the LHS are relativistic corrections that are neglected in a non-relativistic framework. Thus, in the non-relativistic limit, the scalar product (44) reduces to,

$$
(-\mathbf{D} \times(\nabla \times \mathbf{E})-\mathbf{B} \times(\nabla \times \mathbf{H})) \cdot \mathbf{v}=\left(\mathbf{j}_{q} \times \mathbf{B}\right) \cdot \mathbf{v} .
$$

Using the vectorial identities,

$$
-\mathbf{D} \times(\nabla \times \mathbf{E})=-\mathbf{D} \nabla \mathbf{E}-(\nabla \cdot \mathbf{D}) \mathbf{E}+\nabla_{\mathbf{D}} \cdot(\mathbf{D} \odot \mathbf{E}),
$$

and

$$
-\mathbf{B} \times(\boldsymbol{\nabla} \times \mathbf{H})=-\mathbf{B} \nabla \mathbf{H}-(\boldsymbol{\nabla} \cdot \mathbf{B}) \mathbf{H}+\nabla_{\mathbf{B}} \cdot(\mathbf{B} \odot \mathbf{H}),
$$

and Gauss' law (16) and Thomson's law (17), the Eq. (45) is recast as,

$$
\left(\nabla_{\mathbf{D}, \mathbf{B}} \cdot(\mathbf{D} \odot \mathbf{E}+\mathbf{B} \odot \mathbf{H})\right) \cdot \mathbf{v}=\left(q \mathbf{E}+\mathbf{j}_{q} \times \mathbf{B}+\mathbf{D} \nabla \mathbf{E}+\mathbf{B} \nabla \mathbf{H}\right) \cdot \mathbf{v},
$$

where the index $\mathbf{D , B}$ denotes that there is a dot product between the covariant differential operator $\nabla$ and the contravariant electromagnetic vector fields $\mathbf{D}$ and $\mathbf{B}$, respectively.

In order to recast the terms $\mathbf{D} \nabla \mathbf{E}$ and $\mathbf{B} \boldsymbol{\nabla} \mathbf{H}$ on the RHS of Eq. (46) and obtain explicitly Eq. (43), we need to introduce the Legendre transform of the electromagnetic part of the internal energy density $\ell_{u}^{\mathrm{em}}\left(s, n_{A}, \mathbf{E}, \mathbf{H}\right)$. The Legendre transform of the electromagnetic part of the internal energy density $\ell_{u}^{\mathrm{em}}\left(s, n_{A}, \mathbf{E}, \mathbf{H}\right)$ is defined as,

$$
\ell_{u}^{\mathrm{em}}\left(s, n_{A}, \mathbf{E}, \mathbf{H}\right) \equiv \frac{\partial u^{\mathrm{em}}}{\partial \mathbf{D}} \cdot \mathbf{D}+\frac{\partial u^{\mathrm{em}}}{\partial \mathbf{B}} \cdot \mathbf{B}-u^{\mathrm{em}}\left(s, n_{A}, \mathbf{D}, \mathbf{B}\right)
$$

Using the expression (31) for the electromagnetic part of the internal energy density and the differential relations (29), the Legendre transform (47) is found to be,

$$
\ell_{u}^{\mathrm{em}}\left(s, n_{A}, \mathbf{E}, \mathbf{H}\right)=\frac{1}{2}\left(\mathbf{E} \cdot \mathbf{D}\left(s, n_{A}, \mathbf{E}, \mathbf{H}\right)+\mathbf{H} \cdot \mathbf{B}\left(s, n_{A}, \mathbf{E}, \mathbf{H}\right)\right)
$$

where the integrability conditions are,

$$
\begin{aligned}
& \mathbf{D}\left(s, n_{A}, \mathbf{E}, \mathbf{H}\right)=\frac{\partial \ell_{u}^{\mathrm{em}}\left(s, n_{A}, \mathbf{E}, \mathbf{H}\right)}{\partial \mathbf{E}}, \\
& \mathbf{B}\left(s, n_{A}, \mathbf{E}, \mathbf{H}\right)=\frac{\partial \ell_{u}^{\mathrm{em}}\left(s, n_{A}, \mathbf{E}, \mathbf{H}\right)}{\partial \mathbf{H}} .
\end{aligned}
$$

Using the electromagnetic constitutive relations (11), the Legendre transform (48) can be recast as,

$$
\begin{aligned}
\ell_{u}^{\mathrm{em}}\left(s, n_{A}, \mathbf{E}, \mathbf{H}\right)= & \frac{1}{2}\left(\boldsymbol{\varepsilon}\left(s, n_{A}\right) \cdot(\mathbf{E} \odot \mathbf{E})+\boldsymbol{\mu}\left(s, n_{A}\right) \cdot(\mathbf{H} \odot \mathbf{H})\right) \\
& +\left(\boldsymbol{\alpha}\left(s, n_{A}\right) \cdot \boldsymbol{\mu}\left(s, n_{A}\right)\right) \cdot(\mathbf{E} \otimes \mathbf{H}) .
\end{aligned}
$$

The constitutive relations (11) imply that the gradient of the Legendre transform (50) is of the form,

$$
\begin{aligned}
\nabla \ell_{u}^{\mathrm{em}}= & \mathbf{D} \nabla \mathbf{E}+\mathbf{B} \nabla \mathbf{H}+\frac{1}{2}((\mathbf{E} \odot \mathbf{E}) \nabla \boldsymbol{\varepsilon}+(\mathbf{H} \odot \mathbf{H}) \nabla \boldsymbol{\mu}) \\
& +(\mathbf{E} \otimes \mathbf{H}) \nabla(\boldsymbol{\alpha} \cdot \boldsymbol{\mu}) .
\end{aligned}
$$


Substituting the relation (51) into the Eq. (46), the latter reduces to,

$$
\begin{aligned}
& \left(\nabla_{\mathbf{D}, \mathbf{B}} \cdot\left(\mathbf{D} \odot \mathbf{E}+\mathbf{B} \odot \mathbf{H}-\ell_{u}^{\mathrm{em}} \mathbb{1}\right)\right) \cdot \mathbf{v} \\
& \quad=\left(q \mathbf{E}+\mathbf{j}_{q} \times \mathbf{B}-\frac{1}{2}((\mathbf{E} \odot \mathbf{E}) \nabla \boldsymbol{\varepsilon}+(\mathbf{H} \odot \mathbf{H}) \nabla \boldsymbol{\mu})-(\mathbf{E} \otimes \mathbf{H}) \nabla(\boldsymbol{\alpha} \cdot \boldsymbol{\mu})\right) \cdot \mathbf{v} .
\end{aligned}
$$

By identification of the Eqs. (46) and (52), the expressions for the electromagnetic stress tensor $\sigma^{\mathrm{em}}$ and the electromagnetic force density $\mathbf{f}^{\mathrm{em}}$ are found to be,

$$
\begin{aligned}
& \sigma^{\mathrm{em}}=\mathbf{D} \odot \mathbf{E}+\mathbf{B} \odot \mathbf{H}-\frac{1}{2}(\mathbf{E} \cdot \mathbf{D}+\mathbf{H} \cdot \mathbf{B}) \mathbb{1}, \\
& \mathbf{f}^{\mathrm{em}}=q \mathbf{E}+\mathbf{j}_{q} \times \mathbf{B}-\frac{1}{2}((\mathbf{E} \odot \mathbf{E}) \nabla \boldsymbol{\varepsilon}+(\mathbf{H} \odot \mathbf{H}) \nabla \boldsymbol{\mu})-(\mathbf{E} \otimes \mathbf{H}) \nabla(\boldsymbol{\alpha} \cdot \boldsymbol{\mu}) .
\end{aligned}
$$

Using the electromagnetic constitutive relations (6), the definitions (5) of the phenomenological tensors, the vectorial relation,

$$
\nabla \mu^{-1}=-\mu^{2} \nabla \mu
$$

and the weak coupling condition (8), the electromagnetic force density (54) is recast in terms of the intensive electromagnetic fields $\mathbf{E}$ and $\mathbf{B}$ and the susceptibility tensors $\chi_{e}, \chi_{m}$ and $\chi_{\alpha}$ as,

$$
\begin{aligned}
\mathbf{f}^{\mathrm{em}}= & q \mathbf{E}+\mathbf{j}_{q} \times \mathbf{B}-\frac{1}{2} \varepsilon_{0}\left((\mathbf{E} \odot \mathbf{E}) \nabla \chi_{e}+(c \mathbf{B} \odot c \mathbf{B}) \nabla \chi_{m}\right) \\
& -\varepsilon_{0}(\mathbf{E} \otimes c \mathbf{B}) \nabla \chi_{\alpha} .
\end{aligned}
$$

The first and second terms on the RHS of the expression (55) represent the Lorentz force density exerted by the electromagnetic fields $\mathbf{E}$ and $\mathbf{B}$ on the matter fields $q$ and $\mathbf{j}_{q}$. The third and fourth terms represent the ponderomotive force density exerted by the gradient of the matter fields $\chi_{e}, \chi_{m}$ and $\chi_{\alpha}$ on the electromagnetic fields $\mathbf{E}$ and $\mathbf{B}$ [4]. The Lorentz force density represents the action of the electromagnetic fields on the matter fields, and the ponderomotive force densities represent the reaction of the matter fields on the electromagnetic fields. Therefore, the ponderomotive force densities have a negative sign in the expression (55) for the electromagnetic force density $\mathbf{f}^{\mathrm{em}}$.

\subsection{Angular momenta and angular mass continuity equations}

The continuity equation for the total angular momentum density $\boldsymbol{J}$ is given by,

$$
\boldsymbol{j}+(\boldsymbol{\nabla} \cdot \mathbf{v}) \boldsymbol{J}+\nabla \cdot \mathrm{j}_{\boldsymbol{J}}=\mathbf{r} \times \mathbf{f}^{\mathrm{ext}}+\boldsymbol{\tau}^{\mathrm{ext}},
$$

where $\mathbf{r}$ is the position of the centre of mass of the local element of continuous medium. The source density on the RHS of the continuity equation (56) consists of two parts: an orbital part $\mathbf{r} \times \mathbf{f}^{\text {ext }}$ that is due to the torque resulting from the action of the external force density $\mathbf{f}^{\text {ext }}$ and an intrinsic part that is due to an external intrinsic torque density $\tau^{\mathrm{ext}}$.

The total angular momentum density $\boldsymbol{J}$ also consists of two parts: an orbital part $\ell$, that is independent of the intrinsic angular velocity $\omega$, and an intrinsic part $\mathbf{s}$, that is independent of the velocity $\mathbf{v}$ according to,

$$
\boldsymbol{J}\left(s, n_{A}, \mathbf{v}, \boldsymbol{\omega}, \mathbf{D}, \mathbf{A}\right)=\ell\left(s, n_{A}, \mathbf{v}, \mathbf{D}, \mathbf{B}(\mathbf{A})\right)+\mathbf{s}\left(s, n_{A}, \boldsymbol{\omega}, \mathbf{D}, \mathbf{A}\right),
$$

where the magnetic induction field $\mathbf{B}(\mathbf{A})$ is entirely determined by the electromagnetic vector potential $\mathbf{A}$, i.e.,

$$
\mathbf{B}(\mathbf{A})=\nabla \times \mathbf{A} .
$$

The angular momentum current density $\mathrm{j}_{J}$ consists of two parts as well: an orbital part $\mathrm{j}_{\ell}$ and an intrinsic part $\mathrm{j}_{\mathrm{s}}$ according to,

$$
\mathrm{j}_{J}=\mathrm{j}_{\ell}+\mathrm{j}_{\mathrm{s}}
$$


These current densities account for the local change in magnitude of the angular momentum due to the migration of the matter and the time evolution of the electromagnetic fields.

The intrinsic angular momentum density $\mathbf{s}$ is independent of the position $\mathbf{r}$. Thus, the continuity equation for the total angular momentum (56) implies that the continuity equation for the intrinsic angular momentum is of the form,

$$
\dot{\mathbf{s}}+(\nabla \cdot \mathbf{v}) \mathbf{s}-\nabla \cdot \Theta=\tau^{\mathrm{ext}},
$$

where the intrinsic angular stress density pseudotensor $\Theta$ is defined as the opposite of the intrinsic angular momentum current density tensor $j_{s}[5]$, i.e.,

$$
\Theta \equiv-\mathrm{j}_{\mathbf{s}}
$$

Note that the intrinsic angular momentum density s should not be confused with the quantum mechanical "spin density" for two reasons. First, the spin is a genuine quantum mechanical property, which has no classical counterpart. Secondly, in a phenomenological macroscopic theory, the intrinsic rotation of the local rest frame with respect to the local material frame generates a mechanical intrinsic angular momentum density, which is not due to the intrinsic properties of the elementary particles in the local system but to their dynamics.

The intrinsic angular momentum density consists of two parts: a matter part $\mathbf{s}^{\text {mat }}\left(s, n_{A}, \boldsymbol{\omega}\right)$ and an electromagnetic part $\mathbf{s}^{\mathrm{em}}(\mathbf{D}, \mathbf{A})$ according to,

$$
\mathbf{s}\left(s, n_{A}, \boldsymbol{\omega}, \mathbf{D}, \mathbf{A}\right)=\mathbf{s}^{\mathrm{mat}}\left(s, n_{A}, \boldsymbol{\omega}\right)+\mathbf{s}^{\mathrm{em}}(\mathbf{D}, \mathbf{A}),
$$

where

$$
\begin{aligned}
& \mathbf{s}^{\text {mat }}\left(s, n_{A}, \boldsymbol{\omega}\right)=I\left(s, n_{A}\right) \boldsymbol{\omega}, \\
& \mathbf{s}^{\mathrm{em}}(\mathbf{D}, \mathbf{A})=\mathbf{D} \times \mathbf{A} .
\end{aligned}
$$

For a homogeneous sphere of constant infinitesimal radius $d r$, the intrinsic angular mass density $I$ is related to the mass density $m$ by,

$$
I=\frac{2}{5} m d r^{2}
$$

The continuity equation for the mass (41) and the relation (65) implies that the continuity for the intrinsic angular mass is given by,

$$
\dot{I}+(\nabla \cdot \mathbf{v}) I=0 .
$$

Similarly, the intrinsic angular stress density pseudotensor $\Theta$ also consists of two parts: a matter part $\boldsymbol{\Theta}^{\text {mat }}\left(s, n_{A}, \boldsymbol{\omega}\right)$ and an electromagnetic part $\boldsymbol{\Theta}^{\mathrm{em}}\left(s, n_{A}, \boldsymbol{\omega}, \mathbf{D}, \mathbf{A}\right)$ according to,

$$
\boldsymbol{\Theta}\left(s, n_{A}, \boldsymbol{\omega}, \mathbf{D}, \mathbf{A}\right)=\boldsymbol{\Theta}^{\mathrm{mat}}\left(s, n_{A}, \boldsymbol{\omega}\right)+\boldsymbol{\Theta}^{\mathrm{em}}\left(s, n_{A}, \boldsymbol{\omega}, \mathbf{D}, \mathbf{A}\right) .
$$

The relations (62) and (67) imply that the continuity equation (60) can be split according to,

$$
\begin{aligned}
& \dot{\mathbf{s}}^{\mathrm{mat}}+(\boldsymbol{\nabla} \cdot \mathbf{v}) \mathbf{s}^{\mathrm{mat}}-\nabla \cdot \boldsymbol{\Theta}^{\mathrm{mat}}=\boldsymbol{\tau}^{\mathrm{ext}}+\boldsymbol{\tau}^{\mathrm{em}}, \\
& \dot{\mathbf{s}}^{\mathrm{em}}+(\boldsymbol{\nabla} \cdot \mathbf{v}) \mathbf{s}^{\mathrm{em}}-\nabla \cdot \boldsymbol{\Theta}^{\mathrm{em}}=-\boldsymbol{\tau}^{\mathrm{em}},
\end{aligned}
$$

where $\tau^{\mathrm{em}}$ is the electromagnetic torque density exerted by the electromagnetic fields on the matter fields. Note that the electromagnetic torque density $\tau^{\mathrm{em}}$ is an internal torque density for the whole system. However, it is considered as an "external" torque density for the matter subsystem.

Note that in the particular case where the continuity equation (68) is applied at the interface between two different magnetic layers, the external torque density $\boldsymbol{\tau}^{\text {ext }}$ accounts for the transfer of intrinsic angular momentum and is thus related to the classical counterpart of the spin transfer torque.

Using the expression (63) for the momentum density of the matter part and the continuity equation for the intrinsic angular mass, the continuity equation (68) for the intrinsic angular momentum density of the matter part becomes,

$$
I \dot{\omega}=\tau^{\mathrm{ext}}+\tau^{\mathrm{em}}+\nabla \cdot \Theta^{\mathrm{mat}}
$$


which is Newton's second law in intrinsic rotation where $\boldsymbol{\nabla} \cdot \boldsymbol{\Theta}^{\text {mat }}$ is the matter intrinsic torque density.

The local element of continuous medium is an infinitesimal sphere of radius $d r$. Thus, the scalar product $\left(\dot{\mathbf{s}}^{\mathrm{em}}+(\boldsymbol{\nabla} \cdot \mathbf{v}) \mathbf{s}^{\mathrm{em}}\right) \cdot \boldsymbol{\omega}$ in the continuity equation (69) is a relativistic correction since $\omega^{2} d r^{2} / c^{2} \ll 1$. Hence, in a non-relativistic framework, the scalar product of the continuity equation (39) and the intrinsic velocity vector $\omega$ reduces to,

$$
\left(\nabla \cdot \Theta^{\mathrm{em}}\right) \cdot \boldsymbol{\omega}=\tau^{\mathrm{em}} \cdot \boldsymbol{\omega} .
$$

We choose the non-relativistic frame-independent Coulomb gauge,

$$
\nabla \cdot \mathbf{A}=0 .
$$

Using the vectorial identity,

$$
(\boldsymbol{\nabla} \cdot \mathbf{v}) \mathbf{B}-(\mathbf{B} \cdot \boldsymbol{\nabla}) \mathbf{v}=-(\mathbf{v} \cdot \boldsymbol{\nabla}) \mathbf{B}-\nabla \times(\mathbf{v} \times \mathbf{B}),
$$

and the definition (58) of the electromagnetic vector potential $\mathbf{A}$, the Faraday equation (23) can be recast in terms of $\mathbf{A}$ as,

$$
(\boldsymbol{\nabla} \times \mathbf{A})^{\cdot}-(\mathbf{v} \cdot \boldsymbol{\nabla})(\boldsymbol{\nabla} \times \mathbf{A})=-\nabla \times(\mathbf{E}-\mathbf{v} \times(\boldsymbol{\nabla} \times \mathbf{A})) .
$$

Moreover, using the vectorial identities,

$$
(\boldsymbol{\nabla} \times \mathbf{A})^{\cdot}=\nabla \times(\dot{\mathbf{A}}-(\mathbf{v} \cdot \nabla) \mathbf{A})+(\mathbf{v} \cdot \nabla)(\boldsymbol{\nabla} \times \mathbf{A}),
$$

and

$$
-\mathbf{v} \times(\boldsymbol{\nabla} \times \mathbf{A})=-\mathbf{v} \nabla \mathbf{A}+(\mathbf{v} \cdot \nabla) \mathbf{A},
$$

the Faraday equation (73) reduces to,

$$
\nabla \times(\mathbf{E}+\dot{\mathbf{A}}-\mathbf{v} \nabla \mathbf{A})=\mathbf{0}
$$

and admits a solution of the form,

$$
\mathbf{E}+\dot{\mathbf{A}}-\mathbf{v} \nabla \mathbf{A}=-\nabla(\Phi+\mathbf{v} \cdot \mathbf{A}),
$$

where $\Phi$ is the electromagnetic scalar potential in the local material frame. The relation (75) can be recast as,

$$
\mathbf{E}=-\dot{\mathbf{A}}-\mathbf{A} \nabla \mathbf{v}-\nabla \Phi .
$$

In the non-relativistic limit, Maxwell-Ampère's law (22) and Faraday's law (76) imply that the scalar product of the continuity equation (69) and the intrinsic vorticity field $\omega$ can be expressed as,

$$
\begin{aligned}
& \left((\mathbf{D} \times \mathbf{A})^{\cdot}+(\boldsymbol{\nabla} \cdot \mathbf{v})(\mathbf{D} \times \mathbf{A})-((\mathbf{D} \cdot \boldsymbol{\nabla}) \mathbf{v}) \times \mathbf{A}-\mathbf{D} \times((\mathbf{A} \cdot \boldsymbol{\nabla}) \mathbf{v})\right) \cdot \boldsymbol{\omega} \\
& \quad=\left(-\mathbf{A} \times(\boldsymbol{\nabla} \times \mathbf{H})-\mathbf{j}_{q} \times \mathbf{A}-\mathbf{D} \times(\mathbf{E}+\nabla \Phi)\right) \cdot \boldsymbol{\omega},
\end{aligned}
$$

where the terms on the LHS are relativistic corrections that are neglected in a non-relativistic framework. Thus, in the non-relativistic limit, the scalar product (77) reduces to,

$$
(-\mathbf{A} \times(\boldsymbol{\nabla} \times \mathbf{H})) \cdot \boldsymbol{\omega}=\left(\mathbf{j}_{q} \times \mathbf{A}+\mathbf{D} \times(\mathbf{E}+\nabla \Phi)\right) \cdot \boldsymbol{\omega} .
$$

In the Coulomb gauge (72), using the vectorial identities,

$$
-\mathbf{A} \times(\boldsymbol{\nabla} \times \mathbf{H})=-\mathbf{A} \nabla \mathbf{H}-(\boldsymbol{\nabla} \cdot \mathbf{A}) \mathbf{H}+\nabla_{\mathbf{A}} \cdot(\mathbf{A} \odot \mathbf{H}),
$$

and

$$
\mathbf{B} \times \mathbf{H}=-\mathbf{H} \nabla \mathbf{A}-(\boldsymbol{\nabla} \cdot \mathbf{H}) \mathbf{A}+\nabla_{\mathbf{H}} \cdot(\mathbf{H} \odot \mathbf{A}),
$$


and

$$
\mathbf{A} \nabla \mathbf{H}+\mathbf{H} \nabla \mathbf{A}=\nabla \cdot((\mathbf{A} \cdot \mathbf{H}) \mathbb{1})
$$

the Eq. (78) is recast as,

$$
\begin{aligned}
& \left(\nabla_{\mathbf{A}, \mathbf{H}} \cdot(2 \mathbf{A} \odot \mathbf{H}-(\mathbf{A} \cdot \mathbf{H}) \mathbb{1})\right) \cdot \omega \\
& \quad=\left(\mathbf{j}_{q} \times \mathbf{A}+\mathbf{D} \times(\mathbf{E}+\nabla \Phi)+\mathbf{B} \times \mathbf{H}+\mathbf{A}(\nabla \cdot \mathbf{H})\right) \cdot \omega .
\end{aligned}
$$

By identification of the Eqs. (71) and (79), the expressions for the electromagnetic angular stress density pseudotensor $\boldsymbol{\Theta}^{\mathrm{em}}$ and the electromagnetic torque density $\boldsymbol{\tau}^{\mathrm{em}}$ are found to be,

$$
\begin{aligned}
& \boldsymbol{\Theta}^{\mathrm{em}}=2 \mathbf{A} \odot \mathbf{H}-(\mathbf{A} \cdot \mathbf{H}) \mathbb{1}, \\
& \boldsymbol{\tau}^{\mathrm{em}}=\mathbf{j}_{q} \times \mathbf{A}+\mathbf{D} \times(\mathbf{E}+\nabla \Phi)+\mathbf{B} \times \mathbf{H}+\mathbf{A}(\boldsymbol{\nabla} \cdot \mathbf{H}) .
\end{aligned}
$$

The expressions (80) and (81) for $\boldsymbol{\Theta}^{\mathrm{em}}$ and $\boldsymbol{\tau}^{\mathrm{em}}$, respectively, are an extension of the expressions derived by Santarelli [6] in the absence of matter sources. Using the electromagnetic constitutive relations (6) and the definitions (5) of the phenomenological tensors, the electromagnetic torque density (81) is recast in terms of the intensive electromagnetic fields $\mathbf{E}$ and $\mathbf{B}$ and the susceptibility tensors $\chi_{e}, \chi_{m}$ and $\chi_{\alpha}$ as,

$$
\begin{aligned}
\boldsymbol{\tau}^{\mathrm{em}}= & \mathbf{j}_{q} \times \mathbf{A}+\varepsilon_{0}\left(\chi_{e} \cdot \mathbf{E}+\chi_{\alpha} \cdot c \mathbf{B}\right) \times(\mathbf{E}+\nabla \Phi) \\
& +\varepsilon_{0}\left(\chi_{m} \cdot c \mathbf{B}-\chi_{\alpha} \cdot \mathbf{E}\right) \times c \mathbf{B}-\varepsilon_{0} c \mathbf{A} \nabla \cdot\left(\chi_{m} \cdot c \mathbf{B}-\chi_{\alpha} \cdot \mathbf{E}\right) .
\end{aligned}
$$

The first term on the RHS of the expression (81) represents the Lorentz torque density exerted by the electromagnetic field $\mathbf{A}$ on the matter field $\mathbf{j}_{q}$. The second and third terms represent the torques exerted by the electromagnetic fields $\mathbf{E}, \mathbf{B}$ and $\nabla \Phi$ on the matter fields $\chi_{e}, \chi_{m}$ and $\chi_{\alpha}$. The fourth term represents the ponderomotive torque density exerted by the gradient of the matter fields $\chi_{m}$ and $\chi_{\alpha}$ on the electromagnetic fields $\mathbf{E}$ and $\mathbf{B}$ [4]. The first three terms represent the action of the electromagnetic fields on the matter fields, and the last term represents the reaction of the matter fields on the electromagnetic fields. Therefore, the ponderomotive torque density has a negative sign in the expression (82) for the electromagnetic torque density $\boldsymbol{\tau}^{\mathrm{em}}$.

The continuity equation for the orbital angular momentum $\ell$ that is related to the momentum by,

$$
\ell\left(s, n_{A}, \mathbf{v}, \mathbf{D}, \mathbf{B}\right)=\mathbf{r} \times \mathbf{p}\left(s, n_{A}, \mathbf{v}, \mathbf{D}, \mathbf{B}\right),
$$

is obtained by subtracting the continuity equation (60) from the continuity equation (56), i.e.,

$$
\dot{\ell}+(\nabla \cdot \mathbf{v}) \ell+\nabla \cdot j_{\ell}=\mathbf{r} \times \mathbf{f}^{\mathrm{ext}},
$$

which is Newton's second law of motion in rotation.

The vectorial product between the position $\mathbf{r}$ and the continuity equation for the momentum (33) yields,

$$
\mathbf{r} \times \dot{\mathbf{p}}+(\nabla \cdot \mathbf{v}) \ell-\mathbf{r} \times(\nabla \cdot \sigma)=\mathbf{r} \times \mathbf{f}^{\mathrm{ext}} .
$$

Using the time derivative of the relation (83),

$$
\dot{\ell}=\mathbf{v} \times \mathbf{p}+\mathbf{r} \times \dot{\mathbf{p}}
$$

where $\mathbf{v}=\dot{\mathbf{r}}$, the difference between the relations (85) and (84) is given by,

$$
\nabla_{\sigma} \cdot(\mathbf{r} \times \boldsymbol{\sigma})-\mathbf{r} \times(\nabla \cdot \sigma)-\mathbf{v} \times \mathbf{p}=\mathbf{0} .
$$

The definitions of the momentum densities (34)-(36) imply that the vector product,

$$
\mathbf{v} \times \mathbf{p}=\mathbf{v} \times m \mathbf{v}+\mathbf{v} \times(\mathbf{D} \times \mathbf{B}),
$$

is a relativistic correction, which is neglected in a non-relativistic framework. Thus, in the non-relativistic limit, the relation (86) reduces to,

$$
\nabla_{\sigma} \cdot(\mathbf{r} \times \boldsymbol{\sigma})-\mathbf{r} \times(\nabla \cdot \sigma)=\mathbf{0} .
$$


Substituting the vectorial identity,

$$
\nabla_{\sigma} \cdot(\mathbf{r} \times \sigma)=\hat{\sigma}+\mathbf{r} \times(\nabla \cdot \sigma),
$$

where $\hat{\sigma}$ is the stress pseudovector defined as the dual of the antisymmetric part of the stress tensor and written explicitly in components as,

$$
\hat{\sigma}^{i}=\varepsilon^{i j k} \sigma_{j k}
$$

into the relation (87) implies that,

$$
\hat{\boldsymbol{\sigma}}=\mathbf{0}
$$

which means that the stress tensor $\sigma$ is a symmetric rank-2 tensor.

\subsection{Internal energy continuity equation}

The continuity equation for the internal energy density $u$ reads,

$$
\dot{u}+(\nabla \cdot \mathbf{v}) u+\nabla \cdot \mathbf{j}_{u}=\rho_{u} .
$$

Using the continuity equations for the mass (41), the intrinsic angular mass (66) and the energy (26), Newton's second law in translation (42) and Newton's second law in intrinsic rotation (70), the electromagnetic dynamical equations (43) and (71), the splittings (37) and (67), the time derivative of the relation (27) yields,

$$
\dot{e}=\dot{u}+(\nabla \cdot \mathbf{v})(u-e)+(\nabla \cdot \sigma) \cdot \mathbf{v}+(\nabla \cdot \boldsymbol{\Theta}) \cdot \boldsymbol{\omega}+\mathbf{f}^{\text {ext }} \cdot \mathbf{v}+\boldsymbol{\tau}^{\text {ext }} \cdot \boldsymbol{\omega} .
$$

Substituting the relation (91) into the continuity equation (26) for the energy and using the vectorial identities,

$$
\begin{aligned}
& (\nabla \cdot \sigma) \cdot \mathbf{v}=\nabla_{\sigma} \cdot(\sigma \cdot \mathbf{v})-\sigma \cdot(\nabla \odot \mathbf{v}), \\
& (\nabla \cdot \boldsymbol{\Theta}) \cdot \boldsymbol{\omega}=\nabla_{\boldsymbol{\Theta}} \cdot(\boldsymbol{\Theta} \cdot \boldsymbol{\omega})-\boldsymbol{\Theta} \cdot(\boldsymbol{\nabla} \odot \boldsymbol{\omega}),
\end{aligned}
$$

the expression for the internal energy current density $\mathbf{j}_{u}$ and the internal energy source density $\rho_{u}$ is identified, respectively, as,

$$
\begin{aligned}
& \mathbf{j}_{u}=\mathbf{j}_{e}+\boldsymbol{\sigma} \cdot \mathbf{v}+\boldsymbol{\Theta} \cdot \boldsymbol{\omega}, \\
& \rho_{u}=\boldsymbol{\sigma} \cdot(\nabla \odot \mathbf{v})+\boldsymbol{\Theta} \cdot(\nabla \odot \boldsymbol{\omega}),
\end{aligned}
$$

where the indices $\sigma$ or $\Theta$ denote that there is a dot product between the covariant differential operator $\nabla$ and the contravariant components of the stress tensors $\sigma$ and $\Theta$, respectively. Note that,

$$
\mathbf{j}_{W}=-\sigma \cdot \mathbf{v}-\boldsymbol{\Theta} \cdot \boldsymbol{\omega},
$$

represents the work current density due to the matter and electromagnetic fields, and the terms $\sigma \cdot(\nabla \odot \mathbf{v})$ and $\Theta \cdot(\nabla \odot \omega)$ account for the energy loss due to the friction and the rotational friction, respectively.

\section{Time evolution equations}

3.1 Time evolution of the mass density

The mass density $m$ is defined as the density of mass $m_{A}$ carried by the elementary units of chemical substances $A$, i.e.,

$$
m=\sum_{A} n_{A} m_{A}
$$


In order to characterise physically the time evolution of the mass density, we substitute the expression (95) for the chemical composition of the mass density and the continuity equation for the chemical substance (15) into the continuity equation (41) for the mass. The latter then becomes,

$$
\sum_{A}\left(-\left(\nabla \cdot \mathbf{j}_{A}\right) m_{A}+\sum_{a} v_{a A} \omega_{a} m_{A}\right)=0 .
$$

Using the fact that the mass $m_{A}$ is a non-relativistic invariant, i.e.,

$$
\left(\nabla \cdot \mathbf{j}_{A}\right) m_{A}=\nabla \cdot\left(m_{A} \mathbf{j}_{A}\right),
$$

the continuity equation (96) for the mass can be recast as,

$$
\sum_{a, A} v_{a A} \omega_{a} m_{A}-\nabla \cdot\left(\sum_{A} m_{A} \mathbf{j}_{A}\right)=0 .
$$

The continuity equation (97) has to hold for any current flow, which defines a set of frames where the local centre of mass of the matter element is at rest, i.e.,

$$
\sum_{A} m_{A} \mathbf{j}_{A}=\mathbf{0} .
$$

Moreover, it has to hold for every chemical reaction $a$, which implies that

$$
\sum_{A} v_{a A} m_{A}=0,
$$

and means that the chemical reaction $a$ preserves the total mass in the local infinitesimal system.

\subsection{Time evolution of the electric charge density}

The electric charge density $q$ is defined as the density of electric charges $q_{A}$ carried by the elementary units of chemical substances $A$, i.e.,

$$
q=\sum_{A} n_{A} q_{A} .
$$

In order to characterise physically the time evolution of the electric polarisation, we substitute the expression (100) for the chemical composition of the electric charge density and the continuity equation for the chemical substance (15) into the continuity equation (25) for the electric charge. The latter then becomes,

$$
\sum_{A}\left(-\left(\nabla \cdot \mathbf{j}_{A}\right) q_{A}+\sum_{a} v_{a A} \omega_{a} q_{A}\right)+\nabla \cdot \mathbf{j}_{q}=0 .
$$

Using the fact that the electric charge $q_{A}$ is an invariant, i.e.,

$$
\left(\nabla \cdot \mathbf{j}_{A}\right) q_{A}=\nabla \cdot\left(q_{A} \mathbf{j}_{A}\right),
$$

the continuity equation (101) for the electric charge can be recast as,

$$
\sum_{a, A} v_{a A} \omega_{a} q_{A}+\nabla \cdot\left(\mathbf{j}_{q}-\sum_{A} q_{A} \mathbf{j}_{A}\right)=0 .
$$

The continuity equation (102) has to hold for any current flow, which yields an explicit expression for the diffusive electric current density tensor, i.e.,

$$
\mathbf{j}_{q}=\sum_{A} q_{A} \mathbf{j}_{A}
$$

Moreover, it has to hold for every chemical reaction $a$, which implies that

$$
\sum_{A} v_{a A} q_{A}=0
$$

and means that the chemical reaction $a$ preserves the total electric charge in the local infinitesimal system. 
3.3 Time evolution of the matter momentum

The matter momentum density $\mathbf{p}^{\text {mat }}=m \mathbf{v}$ is defined as the density of momentum carried by the elementary units of chemical substances $A$ of velocities $\mathbf{v}_{A}$, i.e.,

$$
\mathbf{p}^{\text {mat }}=\sum_{A} n_{A} m_{A} \mathbf{v}_{A}
$$

It consists of convective part and a diffusive parts according to,

$$
\sum_{A} n_{A} m_{A} \mathbf{v}_{A}=\sum_{A} n_{A} m_{A} \mathbf{v}+\sum_{A} m_{A} \mathbf{j}_{A} .
$$

Thus, using the relation (98), the velocity of the matter element is found to be,

$$
\mathbf{v}=\left(\sum_{A} n_{A} m_{A}\right)^{-1}\left(\sum_{A} n_{A} m_{A} \mathbf{v}_{A}\right)
$$

and represents the velocity of the local centre of mass.

In order to characterise physically the time evolution of the matter momentum, we substitute the expression (35) for the chemical composition of the matter momentum density and the continuity equation for the chemical substance (15) into the continuity equation (38) for the matter momentum. The latter then becomes,

$$
\begin{aligned}
& \sum_{A}\left(n_{A} m_{A} \dot{\mathbf{v}}_{A}-\left(\nabla \cdot \mathbf{j}_{A}\right) m_{A} \mathbf{v}_{A}+\sum_{a} v_{a A} \omega_{a} m_{A} \mathbf{v}_{A}\right) \\
& -\nabla \cdot \boldsymbol{\sigma}^{\mathrm{mat}}=\mathbf{f}^{\mathrm{ext}}+\mathbf{f}^{\mathrm{em}} .
\end{aligned}
$$

The extensivity of the force implies that,

$$
\mathbf{f}^{\mathrm{ext}}+\mathbf{f}^{\mathrm{em}}=\sum_{A}\left(\mathbf{f}_{A}^{\mathrm{ext}}+\mathbf{f}_{A}^{\mathrm{em}}\right)
$$

where $\mathbf{f}_{A}^{\text {ext }}$ represents the non-electromagnetic external force density and $\mathbf{f}_{A}^{\text {em }}$ the electromagnetic force density acting on the chemical substance $A$. In order to determine the explicit expression of $\mathbf{f}_{A}^{\mathrm{em}}$, we express the susceptibility tensors $\chi_{e}, \chi_{m}$ and $\chi_{\alpha}$ in terms of the chemical substances $A$, i.e.,

$$
\chi_{e}=\sum_{A} \chi_{e}^{A}, \quad \chi_{m}=\sum_{A} \chi_{m}^{A}, \quad \chi_{\alpha}=\sum_{A} \chi_{\alpha}^{A}
$$

where $\chi_{e}^{A}, \chi_{m}^{A}$ and $\chi_{\alpha}^{A}$ are the susceptibility tensors of the chemical substance $A$. Substituting the chemical constitutive relations (103), (100) and (110) and the vectorial identity,

$$
\nabla \boldsymbol{\mu}=-\boldsymbol{\mu}^{2} \nabla \boldsymbol{\mu}^{-1}=\varepsilon_{0} c^{2} \boldsymbol{\mu}^{2} \sum_{A} \nabla \chi_{m}^{A}
$$

into the expression (55) of the electromagnetic force density yields, i.e.,

$$
\begin{aligned}
\mathbf{f}_{A}^{\mathrm{em}}= & q_{A}\left(n_{A} \mathbf{E}+\mathbf{j}_{A} \times \mathbf{B}\right)-\frac{1}{2} \varepsilon_{0}\left((\mathbf{E} \odot \mathbf{E}) \nabla \chi_{e}^{A}+(c \mathbf{B} \odot c \mathbf{B}) \nabla \chi_{m}^{A}\right) \\
& -\varepsilon_{0}(\mathbf{E} \otimes c \mathbf{B}) \nabla \chi_{\alpha}^{A} .
\end{aligned}
$$

Using the vectorial identity,

$$
\left(\nabla \cdot \mathbf{j}_{A}\right) \mathbf{v}_{A}=\nabla_{\mathbf{j}} \cdot\left(\mathbf{v}_{A} \odot \mathbf{j}_{A}\right)-\left(\mathbf{j}_{A} \cdot \nabla\right) \mathbf{v}_{A},
$$


and the chemical constitutive equation (109), the continuity equation (108) for the matter momentum is recast as,

$$
\begin{aligned}
& \sum_{A}\left(n_{A} m_{A} \dot{\mathbf{v}}_{A}+m_{A}\left(\mathbf{j}_{A} \cdot \nabla\right) \mathbf{v}_{A}-\mathbf{f}_{A}^{\mathrm{ext}}-\mathbf{f}_{A}^{\mathrm{em}}\right) \\
& \quad+\sum_{a, A} v_{a A} \omega_{a} m_{A} \mathbf{v}_{A}-\nabla_{\mathbf{j}} \cdot\left(\boldsymbol{\sigma}^{\mathrm{mat}}+\sum_{A} m_{A} \mathbf{v}_{A} \odot \mathbf{j}_{A}\right)=\mathbf{0},
\end{aligned}
$$

where the index $\mathbf{j}$ denotes that there is a dot product between the covariant differential operator $\nabla$ and the contravariant current density $\mathbf{j}_{A}$.

The matter stress tensor $\sigma^{\text {mat }}$ is split into a reversible part due to the pressure $P$ and an irreversible part $\tilde{\sigma}^{\text {mat }}$ according to,

$$
\sigma^{\text {mat }}=-P \mathbb{1}+\tilde{\sigma}^{\text {mat }},
$$

which implies that the continuity equation (112) is recast as,

$$
\begin{aligned}
& \sum_{A}\left(n_{A} m_{A} \dot{\mathbf{v}}_{A}+m_{A}\left(\mathbf{j}_{A} \cdot \nabla\right) \mathbf{v}_{A}-\mathbf{f}_{A}^{\mathrm{ext}}-\mathbf{f}_{A}^{\mathrm{em}}\right)+\nabla P \\
& \quad+\sum_{a, A} v_{a A} \omega_{a} m_{A} \mathbf{v}_{A}-\nabla_{\mathbf{j}} \cdot\left(\tilde{\boldsymbol{\sigma}}^{\mathrm{mat}}+\sum_{A} m_{A} \mathbf{v}_{A} \odot \mathbf{j}_{A}\right)=\mathbf{0} .
\end{aligned}
$$

The continuity equation (114) has to hold for any current flow, which yields an explicit expression for the irreverible part of the matter stress tensor, i.e.,

$$
\tilde{\boldsymbol{\sigma}}^{\text {mat }}=-\sum_{A} m_{A} \mathbf{v}_{A} \odot \mathbf{j}_{A}
$$

Moreover, every chemical reaction preserves the total momentum in the local infinitesimal system, i.e.,

$$
\sum_{A} v_{a A} m_{A} \mathbf{v}_{A}=\mathbf{0}
$$

Taking into account the fact that the pressure $P$ of the continuous medium is the sum of the partial pressures $P_{A}$ due to the different chemical substances $A$, i.e.,

$$
P=\sum_{A} P_{A}
$$

and using the conditions (115) and (116), the continuity equation (114) reduces to,

$$
\sum_{A}\left(n_{A} m_{A} \dot{\mathbf{v}}_{A}+m_{A}\left(\mathbf{j}_{A} \cdot \nabla\right) \mathbf{v}_{A}-\mathbf{f}_{A}^{\mathrm{ext}}-\mathbf{f}_{A}^{\mathrm{em}}+\nabla P_{A}\right)=\mathbf{0},
$$

which describes the local time evolution of all the chemical substances.

In order to establish the local time evolution of a specific chemical substance $A$ in the presence of other chemical substances $B$, we need to take explicitly into account the local internal force densities $\mathbf{f}_{B \rightarrow A}^{\text {int }}$ exerted by the chemical substances $B$ on the chemical substance $A$. These force densities do not change the local internal energy of the system, which means that they are the densities of conservative forces accounting for elastic collisions or scattering. Newton's third law implies that the sum of the internal force densities over all the chemical substances vanishes, i.e.,

$$
\sum_{A, B} \mathbf{f}_{B \rightarrow A}^{\mathrm{int}}=\mathbf{0}
$$

The Eqs. (118) and (119) imply that the local time evolution of a specific chemical substance $A$ is given by,

$$
n_{A} m_{A} \dot{\mathbf{v}}_{A}=\mathbf{f}_{A}^{\mathrm{ext}}+\mathbf{f}_{A}^{\mathrm{em}}-\nabla P_{A}-m_{A}\left(\mathbf{j}_{A} \cdot \nabla\right) \mathbf{v}_{A}+\sum_{B} \mathbf{f}_{B \rightarrow A}^{\mathrm{int}}
$$

where the first, second, third and fourth terms on the RHS describe, respectively, the action of the external and electromagnetic forces, of the pressure gradient and of the transport, and the last term describes the action of the internal forces due to the local interaction of the chemical substance $A$ with the other chemical substances $B$. 
3.4 Time evolution of the intrinsic matter angular momentum

The matter intrinsic angular momentum density $\mathbf{s}^{\text {mat }}=I \omega$ is defined as the density of intrinsic angular momentum carried by the elementary units of chemical substances $A$ of intrinsic angular velocities $\omega_{A}$, i.e.,

$$
\mathbf{s}^{\mathrm{mat}}=\sum_{A} n_{A} I_{A} \omega_{A}
$$

where $n_{A} I_{A}$ represent the intrinsic angular mass density of the elementary units of chemical substance $A$, and the relations (65) and (95) imply that

$$
n_{A} I_{A}=\frac{2}{5} n_{A} m_{A} d r^{2}
$$

The matter intrinsic angular momentum density $\mathbf{s}^{\text {mat }}$ consists of convective and diffusive parts according to,

$$
\sum_{A} n_{A} I_{A} \omega_{A}=\sum_{A} n_{A} I_{A} \omega+\sum_{A} I_{A} \boldsymbol{\Omega}_{A}^{\mathrm{m}},
$$

where the sum of the diffusive matter intrinsic angular momenta of all the chemical substances $A$ vanish, i.e.,

$$
\sum_{A} I_{A} \boldsymbol{\Omega}_{A}^{\mathrm{m}}=\mathbf{0}
$$

which defines a set of frames where the matter has no average intrinsic rotational motion. Thus, the intrinsic angular velocity of the matter element is found to be,

$$
\omega=\left(\sum_{A} n_{A} I_{A}\right)^{-1}\left(\sum_{A} n_{A} I_{A} \omega_{A}\right),
$$

and represents the average intrinsic angular velocity around the local centre of mass.

In order to characterise physically the time evolution of the matter intrinsic angular momentum, we substitute the expression (63) for the chemical composition of the matter intrinsic angular momentum density and the continuity equation for the chemical substance (15) into the continuity equation (68) for the intrinsic angular momentum. The latter then becomes,

$$
\begin{aligned}
& \sum_{A}\left(n_{A} I_{A} \dot{\boldsymbol{\omega}}_{A}-\left(\nabla \cdot \mathbf{j}_{A}\right) I_{A} \omega_{A}+\sum_{a} v_{a A} \omega_{a} I_{A} \omega_{A}\right) \\
& -\nabla \cdot \boldsymbol{\Theta}=\boldsymbol{\tau}^{\mathrm{ext}}+\boldsymbol{\tau}^{\mathrm{em}} .
\end{aligned}
$$

The extensivity of the torque implies that,

$$
\boldsymbol{\tau}^{\mathrm{ext}}+\boldsymbol{\tau}^{\mathrm{em}}=\sum_{A}\left(\boldsymbol{\tau}_{A}^{\mathrm{ext}}+\boldsymbol{\tau}_{A}^{\mathrm{em}}\right)
$$

where $\boldsymbol{\tau}_{A}^{\text {ext }}$ represents the non-electromagnetic external torque density and $\boldsymbol{\tau}_{A}^{\text {em }}$ the electromagnetic torque density acting on the chemical substance $A$. Substituting the chemical constitutive relations (103) and (110) into the expression (82) of the electromagnetic torque density yields, i.e.,

$$
\begin{aligned}
\boldsymbol{\tau}_{A}^{\mathrm{em}}= & q_{A} \mathbf{j}_{A} \times \mathbf{A}+\varepsilon_{0}\left(\chi_{e}^{A} \cdot \mathbf{E}+\chi_{\alpha}^{A} \cdot c \mathbf{B}\right) \times(\mathbf{E}+\nabla \Phi) \\
& +\varepsilon_{0}\left(\chi_{m}^{A} \cdot c \mathbf{B}-\chi_{\alpha}^{A} \cdot \mathbf{E}\right) \times c \mathbf{B}-\varepsilon_{0} c \mathbf{A} \nabla \cdot\left(\chi_{m}^{A} \cdot c \mathbf{B}-\chi_{\alpha}^{A} \cdot \mathbf{E}\right)
\end{aligned}
$$

Using the vectorial identity,

$$
\left(\nabla \cdot \mathbf{j}_{A}\right) \omega_{A}=\nabla_{\mathbf{j}} \cdot\left(\omega_{A} \odot \mathbf{j}_{A}\right)-\left(\mathbf{j}_{A} \cdot \nabla\right) \boldsymbol{\omega}_{A},
$$


and the chemical constitutive equation (127), the continuity equation (126) for the intrinsic angular momentum is recast as,

$$
\begin{aligned}
& \sum_{A}\left(n_{A} I_{A} \dot{\omega}_{A}+I_{A}\left(\mathbf{j}_{A} \cdot \nabla\right) \omega_{A}-\tau_{A}^{\mathrm{ext}}-\boldsymbol{\tau}_{A}^{\mathrm{em}}\right) \\
& \quad+\sum_{a, A} v_{a A} \omega_{a} I_{A} \omega_{A}-\nabla_{\mathbf{j}} \cdot\left(\boldsymbol{\Theta}+\sum_{A} I_{A} \omega_{A} \odot \mathbf{j}_{A}\right)=\mathbf{0} .
\end{aligned}
$$

The continuity equation (129) has to hold for any current flow, which yields an explicit expression for the irreversible part of the matter intrinsic angular stress tensor, i.e.,

$$
\Theta^{\text {mat }}=-\sum_{A} I_{A} \omega_{A} \odot \mathbf{j}_{A}
$$

Moreover, every chemical reaction preserves the total intrinsic angular momentum in the local infinitesimal system, i.e.,

$$
\sum_{A} v_{a A} I_{A} \omega_{A}=\mathbf{0}
$$

The conditions (130) and (131) imply that the continuity equation (129) reduces to,

$$
\sum_{A}\left(n_{A} I_{A} \dot{\boldsymbol{\omega}}_{A}+I_{A}\left(\mathbf{j}_{A} \cdot \nabla\right) \boldsymbol{\omega}_{A}-\boldsymbol{\tau}_{A}^{\mathrm{ext}}-\boldsymbol{\tau}_{A}^{\mathrm{em}}\right)=\mathbf{0},
$$

which describes the local intrinsic time evolution of all the chemical substances.

In order to establish the local intrinsic time evolution of a specific chemical substance $A$ in the presence of other chemical substances $B$, we need to take explicitly into account the local internal intrinsic torque densities $\tau_{B \rightarrow A}^{\text {int }}$ exerted by the chemical substances $B$ on the chemical substance $A$. These torque densities do not change the local internal energy of the system, which means that they are the densities of conservative torques accounting for elastic collisions or scattering. Newton's third law in intrinsic rotation implies that the sum of the internal torque densities over all the chemical substances vanishes, i.e.,

$$
\sum_{A, B} \boldsymbol{\tau}_{B \rightarrow A}^{\mathrm{int}}=\mathbf{0}
$$

The Eqs. (132) and (133) imply that the local intrinsic time evolution of a specific chemical substance $A$ is given by,

$$
n_{A} I_{A} \dot{\boldsymbol{\omega}}_{A}=\boldsymbol{\tau}_{A}^{\mathrm{ext}}+\boldsymbol{\tau}_{A}^{\mathrm{em}}-I_{A}\left(\mathbf{j}_{A} \cdot \nabla\right) \omega_{A}+\sum_{B} \boldsymbol{\tau}_{B \rightarrow A}^{\mathrm{int}},
$$

where the first, second and third terms on the RHS describe, respectively, the action of the external torques and of the transport, and the last term describes the action of the internal torques due to the local interaction of the chemical substance $A$ with the other chemical substances $B$.

\subsection{Energy balance}

In order to determine the internal energy balance, we take the time derivative of the internal energy density field $u\left(s, n_{A}, \mathbf{D}, \mathbf{B}\right)$ of the system $\Sigma$, i.e.,

$$
\dot{u}=\frac{\partial u}{\partial s} \dot{s}+\sum_{A} \frac{\partial u}{\partial n_{A}} \dot{n}_{A}+\frac{\partial u}{\partial \mathbf{D}} \cdot \dot{\mathbf{D}}+\frac{\partial u}{\partial \mathbf{B}} \cdot \dot{\mathbf{B}} .
$$


The local thermodynamic system is assumed to be at equilibrium. Thus, we can define the intensive fields temperature $T\left(s, n_{A}, \mathbf{D}, \mathbf{B}\right)$ and chemical potential $\mu_{A}\left(s, n_{A}, \mathbf{D}, \mathbf{B}\right)$ of the substance $A$ as the conjugate of the extensive state scalar fields densities $s$ and $n_{A}$ respectively, i.e.,

$$
\begin{gathered}
T\left(s, n_{A}, \mathbf{D}, \mathbf{B}\right) \equiv \frac{\partial u\left(s, n_{A}, \mathbf{D}, \mathbf{B}\right)}{\partial s}, \\
\mu_{A}\left(s, n_{A}, \mathbf{D}, \mathbf{B}\right) \equiv \frac{\partial u\left(s, n_{A}, \mathbf{D}, \mathbf{B}\right)}{\partial n_{A}} .
\end{gathered}
$$

In order to determine the temperature of the system in terms of the electromagnetic fields, we explicitly substitute the expressions (28) and (30) for the internal energy density $u\left(s, n_{A}, \mathbf{D}, \mathbf{B}\right)$ into the definition of the temperature (136), i.e.,

$$
\begin{aligned}
T\left(s, n_{A}, \mathbf{D}, \mathbf{B}\right)= & T\left(s, n_{A}, \mathbf{0}, \mathbf{0}\right)+\frac{1}{2}\left(\frac{\partial \boldsymbol{\varepsilon}^{-1}}{\partial s} \cdot(\mathbf{D} \odot \mathbf{D})+\frac{\partial \boldsymbol{\mu}^{-1}}{\partial s} \cdot(\mathbf{B} \odot \mathbf{B})\right) \\
& -\left(\frac{\partial \boldsymbol{\varepsilon}^{-1}}{\partial s} \cdot \boldsymbol{\alpha}+\boldsymbol{\varepsilon}^{-1} \cdot \frac{\partial \boldsymbol{\alpha}}{\partial s}\right) \cdot(\mathbf{D} \otimes \mathbf{B}) .
\end{aligned}
$$

The definitions (5) and (110) of the phenomenological tensors yield the identities,

$$
\begin{aligned}
& \frac{\partial \boldsymbol{\varepsilon}^{-1}}{\partial s}=-\frac{\partial \boldsymbol{\varepsilon}}{\partial s} \cdot \boldsymbol{\varepsilon}^{-2}=-\varepsilon_{0}\left(\sum_{A} \frac{\partial \chi_{e}^{A}}{\partial s}\right) \cdot \boldsymbol{\varepsilon}^{-2}, \\
& \frac{\partial \boldsymbol{\mu}^{-1}}{\partial s}=-\varepsilon_{0} c^{2}\left(\sum_{A} \frac{\partial \chi_{m}^{A}}{\partial s}\right) \\
& \frac{\partial \boldsymbol{\alpha}}{\partial s}=\varepsilon_{0} c\left(\sum_{A} \frac{\partial \chi_{\alpha}^{A}}{\partial s}\right) .
\end{aligned}
$$

Using the electromagnetic constitutive relations (6) and the identities (138) under the condition (8), the expression for the temperature (137) is recast as,

$$
\begin{aligned}
& T\left(s, n_{A}, \mathbf{D}, \mathbf{B}\right)=T\left(s, n_{A}, \mathbf{0}, \mathbf{0}\right) \\
& -\sum_{A} \varepsilon_{0}\left(\frac{1}{2} \frac{\partial \chi_{e}^{A}}{\partial s} \cdot(\mathbf{E} \odot \mathbf{E})+\frac{1}{2} \frac{\partial \chi_{m}^{A}}{\partial s} \cdot(c \mathbf{B} \odot c \mathbf{B})+\frac{\partial \chi_{\alpha}^{A}}{\partial s} \cdot(\mathbf{E} \otimes c \mathbf{B})\right) .
\end{aligned}
$$

In order to determine the chemical potential of the system in terms of the electromagnetic fields, we explicitly substitute the expressions (28) and (30) for the internal energy density $u\left(s, n_{A}, \mathbf{D}, \mathbf{B}\right)$ into the definition of the chemical potential (136), i.e.,

$$
\begin{aligned}
\mu_{A}\left(s, n_{A}, \mathbf{D}, \mathbf{B}\right)= & \mu_{A}\left(s, n_{A}, \mathbf{0}, \mathbf{0}\right)+\frac{1}{2}\left(\frac{\partial \boldsymbol{\varepsilon}^{-1}}{\partial n_{A}} \cdot(\mathbf{D} \odot \mathbf{D})+\frac{\partial \boldsymbol{\mu}^{-1}}{\partial n_{A}} \cdot(\mathbf{B} \odot \mathbf{B})\right) \\
& -\left(\frac{\partial \boldsymbol{\varepsilon}^{-1}}{\partial n_{A}} \cdot \boldsymbol{\alpha}+\boldsymbol{\varepsilon}^{-1} \cdot \frac{\partial \boldsymbol{\alpha}}{\partial n_{A}}\right) \cdot(\mathbf{D} \otimes \mathbf{B}) .
\end{aligned}
$$

The definitions (5) and (110) of the phenomenological tensors yield the identities,

$$
\begin{aligned}
& \frac{\partial \boldsymbol{\varepsilon}^{-1}}{\partial n_{A}}=-\frac{\partial \boldsymbol{\varepsilon}}{\partial n_{A}} \cdot \boldsymbol{\varepsilon}^{-2}=-\varepsilon_{0} \frac{\partial \chi_{e}^{A}}{\partial n_{A}} \cdot \boldsymbol{\varepsilon}^{-2}, \\
& \frac{\partial \boldsymbol{\mu}^{-1}}{\partial n_{A}}=-\varepsilon_{0} c^{2} \frac{\partial \chi_{m}^{A}}{\partial n_{A}} \\
& \frac{\partial \boldsymbol{\alpha}}{\partial n_{A}}=\varepsilon_{0} c \frac{\partial \chi_{\alpha}^{A}}{\partial n_{A}} .
\end{aligned}
$$


Using the electromagnetic constitutive relations (6) and the identities (141) under the condition (8), the expression for the temperature (140) is recast as,

$$
\begin{aligned}
& \mu_{A}\left(s, n_{A}, \mathbf{D}, \mathbf{B}\right)=\mu_{A}\left(s, n_{A}, \mathbf{0}, \mathbf{0}\right) \\
& -\varepsilon_{0}\left(\frac{1}{2} \frac{\partial \chi_{e}^{A}}{\partial n_{A}} \cdot(\mathbf{E} \odot \mathbf{E})+\frac{1}{2} \frac{\partial \chi_{m}^{A}}{\partial n_{A}} \cdot(c \mathbf{B} \odot c \mathbf{B})+\frac{\partial \chi_{\alpha}^{A}}{\partial n_{A}} \cdot(\mathbf{E} \otimes c \mathbf{B})\right) .
\end{aligned}
$$

Using the relations (29) and (136), the time derivative of the internal energy density (135) is recast as,

$$
\dot{u}=T \dot{s}+\sum_{A} \mu_{A} \dot{n}_{A}+\mathbf{E} \cdot \dot{\mathbf{D}}+\mathbf{H} \cdot \dot{\mathbf{B}} .
$$

The first law or energy balance is given by the continuity equation for the internal energy (90). Using the continuity equations for the entropy (14) and the chemical substance $A$ (15), Maxwell-Ampère's law (22), Faraday's law (23) and the internal energy source density (93), the continuity equation for the internal energy (90) is recast as,

$$
\begin{aligned}
& T\left(\rho_{s}-(\nabla \cdot \mathbf{v}) s-\nabla \cdot \mathbf{j}_{s}\right)+\sum_{A} \mu_{A}\left(\sum_{a} v_{a A} \omega_{a}-(\nabla \cdot \mathbf{v}) n_{A}-\nabla \cdot \mathbf{j}_{A}\right) \\
& \quad+\mathbf{E} \cdot\left(\nabla \times \mathbf{H}-(\nabla \cdot \mathbf{v}) \mathbf{D}+(\mathbf{D} \cdot \nabla) \mathbf{v}-\mathbf{j}_{q}\right) \\
& \quad+\mathbf{H} \cdot(-\nabla \times \mathbf{E}-(\nabla \cdot \mathbf{v}) \mathbf{B}+(\mathbf{B} \cdot \nabla) \mathbf{v}) \\
& \quad+(\nabla \cdot \mathbf{v}) u+\nabla \cdot \mathbf{j}_{u}=\boldsymbol{\sigma} \cdot(\nabla \odot \mathbf{v})+\boldsymbol{\Theta} \cdot(\nabla \odot \omega) .
\end{aligned}
$$

Moreover, using the constitutive electromagnetic relations (10) and the vectorial identities,

$$
\begin{aligned}
& T\left(\nabla \cdot \mathbf{j}_{s}\right)=\nabla \cdot\left(T \mathbf{j}_{s}\right)-\mathbf{j}_{s} \cdot \nabla T, \\
& \sum_{A} \mu_{A}\left(\nabla \cdot \mathbf{j}_{A}\right)=\nabla \cdot\left(\sum_{A} \mu_{A} \mathbf{j}_{A}\right)-\sum_{A} \mathbf{j}_{A} \cdot \nabla \mu_{A}, \\
& \mathbf{H} \cdot(\nabla \times \mathbf{E})-\mathbf{E} \cdot(\nabla \times \mathbf{H})=\nabla \cdot(\mathbf{E} \times \mathbf{H}),
\end{aligned}
$$

and expressing the chemical dissipation in terms of the chemical affinities $\mathcal{A}_{a}$, i.e.,

$$
\mathcal{A}_{a}=\sum_{A} v_{a A}\left(-\mu_{A}\right),
$$

the internal energy balance equation (144) becomes,

$$
\begin{aligned}
& \left(u-T s-\sum_{A} \mu_{A} n_{A}-\mathbf{E} \cdot \mathbf{D}-\mathbf{H} \cdot \mathbf{B}\right)(\nabla \cdot \mathbf{v}) \\
& \quad+\nabla \cdot\left(\mathbf{j}_{u}-T \mathbf{j}_{s}-\sum_{A} \mu_{A} \mathbf{j}_{A}-\mathbf{E} \times \mathbf{H}\right) \\
& \quad+T \rho_{s}-\sum_{a} \omega_{a} \mathcal{A}_{a}+\mathbf{j}_{s} \cdot \nabla T+\sum_{A} \mathbf{j}_{A} \cdot \nabla \mu_{A}-\mathbf{j}_{q} \cdot \mathbf{E} \\
& \quad-\sigma \cdot(\nabla \odot \mathbf{v})-\boldsymbol{\Theta} \cdot(\nabla \odot \boldsymbol{\omega})+(\mathbf{E}(\mathbf{D} \cdot \nabla)+\mathbf{H}(\mathbf{B} \cdot \nabla)) \cdot \mathbf{v}=0 .
\end{aligned}
$$

The electromagnetic dissipation term in (146) is recast in terms of the chemical current densities $\mathbf{j}_{A}$ using (103), i.e.,

$$
\mathbf{j}_{q} \cdot \mathbf{E}=\sum_{A} q_{A} \mathbf{j}_{A} \cdot \mathbf{E}
$$


Moreover, using the definition (53) of the electromagnetic stress density tensor and the relation (37), the vectorial identity,

$$
(\mathbf{E}(\mathbf{D} \cdot \nabla)+\mathbf{H}(\mathbf{B} \cdot \nabla)) \cdot \mathbf{v}=(\mathbf{D} \odot \mathbf{E}+\mathbf{B} \odot \mathbf{H}) \cdot(\nabla \odot \mathbf{v})
$$

is recast as,

$$
\begin{aligned}
& (\mathbf{E}(\mathbf{D} \cdot \nabla)+\mathbf{H}(\mathbf{B} \cdot \nabla)) \cdot \mathbf{v}=\left(\sigma-\sigma^{\mathrm{mat}}\right) \cdot(\nabla \odot \mathbf{v}) \\
& +\frac{1}{2}(\mathbf{E} \cdot \mathbf{D}+\mathbf{H} \cdot \mathbf{B})(\boldsymbol{\nabla} \cdot \mathbf{v}) .
\end{aligned}
$$

Using the identity (147), the expression for the electromagnetic part of the internal energy density (31) and the fact that the scalar product $\Theta^{\mathrm{em}} \cdot(\nabla \odot \omega)$ is a relativistic correction, the internal energy balance equation (146) becomes,

$$
\begin{aligned}
& \left(u-T s-\sum_{A} \mu_{A} n_{A}-\frac{1}{2}(\mathbf{E} \cdot \mathbf{D}+\mathbf{H} \cdot \mathbf{B})\right)(\nabla \cdot \mathbf{v}) \\
& +\nabla \cdot\left(\mathbf{j}_{u}-T \mathbf{j}_{s}-\sum_{A} \mu_{A} \mathbf{j}_{A}-\mathbf{E} \times \mathbf{H}\right) \\
& +T \rho_{s}-\sum_{a} \omega_{a} \mathcal{A}_{a}+\mathbf{j}_{s} \cdot \nabla T+\sum_{A} \mathbf{j}_{A} \cdot\left(\nabla \mu_{A}-q_{A} \mathbf{E}\right) \\
& -\sigma^{\text {mat }} \cdot(\nabla \odot \mathbf{v})-\boldsymbol{\Theta}^{\text {mat }} \cdot(\nabla \odot \omega)=0 .
\end{aligned}
$$

From the expressions (115), (130) for the momentum and intrinsic angular momentum stress tensors of the matter, we obtain the identities,

$$
\begin{aligned}
& \tilde{\boldsymbol{\sigma}}^{\mathrm{mat}} \cdot(\nabla \odot \mathbf{v})=\sum_{A} \mathbf{j}_{A} \cdot\left(-m_{A} \mathbf{v}_{A} \nabla \mathbf{v}\right), \\
& \boldsymbol{\Theta}^{\mathrm{mat}} \cdot(\nabla \odot \boldsymbol{\omega})=\sum_{A} \mathbf{j}_{A} \cdot\left(-I_{A} \boldsymbol{\omega}_{A} \nabla \omega\right) .
\end{aligned}
$$

Using the splitting (113) of the stress tensor into a reversible and an irreversible part, and the identities (150), the internal energy balance equation (149) becomes,

$$
\begin{aligned}
& \left(u-T s+P-\sum_{A} \mu_{A} n_{A}-\frac{1}{2}(\mathbf{E} \cdot \mathbf{D}+\mathbf{H} \cdot \mathbf{B})\right)(\nabla \cdot \mathbf{v}) \\
& +\nabla \cdot\left(\mathbf{j}_{u}-T \mathbf{j}_{s}-\sum_{A} \mu_{A} \mathbf{j}_{A}-\mathbf{E} \times \mathbf{H}\right) \\
& +T \rho_{s}-\sum_{a} \omega_{a} \mathcal{A}_{a}-\mathbf{j}_{s} \cdot(-\nabla T) \\
& -\sum_{A} \mathbf{j}_{A} \cdot\left(-\nabla \mu_{A}+q_{A} \mathbf{E}-m_{A} \mathbf{v}_{A} \nabla \mathbf{v}-I_{A} \omega_{A} \nabla \omega\right)=0 .
\end{aligned}
$$

\subsection{Thermostatic equation, reversible and irreversible thermodynamic equations}

The internal energy balance equation (151) has to hold locally for all flows. This implies that the terms in the first brackets have to vanish, which yields the thermostatic equilibrium equation for the continuous medium, i.e.,

$$
u=T s-P+\sum_{A} \mu_{A} n_{A}+\frac{1}{2}(\mathbf{E} \cdot \mathbf{D}+\mathbf{H} \cdot \mathbf{B}) .
$$


Similarly, the internal energy balance equation (151) has to hold locally for all currents. This implies that the terms in the second brackets have to vanish, which yields the reversible thermodynamic evolution equation for the continuous medium, i.e.,

$$
\mathbf{j}_{u}=T \mathbf{j}_{s}+\sum_{A} \mu_{A} \mathbf{j}_{A}+\mathbf{E} \times \mathbf{H}
$$

The mechanical work current density $\mathbf{j}_{W}$, the heat current density $\mathbf{j}_{Q}$, the chemical energy current density $\mathbf{j}_{\mathrm{CH}}$ and the electromagnetic energy current density $\mathbf{j}_{\mathrm{EM}}$ satisfy the local energy balance,

$$
\mathbf{j}_{e}=\mathbf{j}_{W}+\mathbf{j}_{u}=\mathbf{j}_{W}+\mathbf{j}_{Q}+\mathbf{j}_{\mathrm{CH}}+\mathbf{j}_{\mathrm{EM}},
$$

and are found to be, respectively,

$$
\begin{aligned}
& \mathbf{j}_{W}=-\mathbf{v} \cdot \boldsymbol{\sigma}-\omega \cdot \boldsymbol{\Theta} \quad \text { and } \quad \mathbf{j}_{Q}=T \mathbf{j}_{S}, \\
& \mathbf{j}_{\mathrm{CH}}=\sum_{A} \mu_{A} \mathbf{j}_{A} \quad \text { and } \quad \mathbf{j}_{\mathrm{EM}}=\mathbf{E} \times \mathbf{H} .
\end{aligned}
$$

Finally, the thermostatic equilibrium equation (152) and the reversible thermodynamic evolution equation (153) imply that the internal energy balance equation (151) yields the irreversible thermodynamic evolution equation for the continuous medium, i.e.,

$$
\begin{aligned}
\rho_{s}=\frac{1}{T}\left\{\sum_{a} \omega_{a} \mathcal{A}_{a}+\mathbf{j}_{s} \cdot(-\nabla T)\right. \\
\left.\quad+\sum_{A} \mathbf{j}_{A} \cdot\left(-\nabla \mu_{A}+q_{A} \mathbf{E}-m_{A} \mathbf{v}_{A} \nabla \mathbf{v}-I_{A} \omega_{A} \nabla \omega\right)\right\} .
\end{aligned}
$$

\section{Thermodynamical phenomenology}

\subsection{Linear phenomenological relations and Onsager matrix elements}

In order to deduce the linear phenomenological relations, we extend the approach developed by Onsager [7,8] to include intrinsic rotations. The expression (156) can be formally split into scalar and vectorial parts, which are irreducible representations of the Euclidean group and have different symmetries. Thus, the entropy source density (156) is expressed formally as,

$$
\rho_{s}=\frac{1}{T}\left\{\sum_{a} \omega_{a} \mathcal{A}_{a}+\sum_{\alpha} \mathbf{j}_{\alpha} \cdot \mathbf{F}_{\alpha}\right\},
$$

where $\omega_{a}$ is a scalar chemical reaction rate density, $\mathcal{A}_{a}$ is a scalar chemical affinity, $\mathbf{j}_{\alpha}$ is a vectorial current density, $\mathbf{F}_{\alpha}$ is a vectorial force.

In the relation (157), there are two types of vectorial current densities and forces (i.e. $\alpha \in\left\{{ }_{s},{ }_{A}\right\}$ ). First, there is a thermal current density $\mathbf{j}_{s}$ and a dissipative thermal force $\mathbf{F}_{s}=-\nabla T$. Second, there are current densities $\mathbf{j}_{A}$ for the chemical substances $A$ and dissipative forces $\mathbf{F}_{A}$ acting on these substances. The expression for the forces $\mathbf{F}_{A}$ is given by the dissipation relation (156), i.e.,

$$
\mathbf{F}_{A}=-\nabla \mu_{A}+q_{A} \mathbf{E}-m_{A} \mathbf{v}_{A} \nabla \mathbf{v}-I_{A} \omega_{A} \nabla \omega,
$$

where the first term on the RHS is the chemical force, the second term is the Coulomb force, and the third and the fourth terms are the viscous forces.

The local expression of the second law (14) requires the entropy source density to be locally positive definite, i.e., $\rho_{s} \geqslant 0$. Using Onsager's approach, in the neighbourhood of a local thermodynamic state where the scalar affinities $\mathcal{A}_{a}$ and the vectorial forces $\mathbf{F}_{\alpha}$ are sufficiently small, the entropy source density can be expressed formally as the sum of quadratic forms of $\mathcal{A}_{a}$ and $\mathbf{F}_{\alpha}$, which ensures that it is positive definite, i.e.,

$$
\rho_{s}=\frac{1}{T}\left(\sum_{a, b} L_{a b} \mathcal{A}_{a} \mathcal{A}_{b}+\sum_{\alpha, \beta} \mathrm{L}_{\alpha \beta} \cdot\left(\mathbf{F}_{\alpha} \odot \mathbf{F}_{\beta}\right)\right) \geqslant 0,
$$


where the phenomenological components are the Onsager matrix elements, which are of two different types: scalars $L_{\alpha \beta}$ and rank-2 tensors $\mathrm{L}_{\alpha \beta}$. The symmetries of the Onsager matrices are given by the Onsager reciprocity relations, i.e.,

$$
\begin{aligned}
& L_{a b}\left(s, n_{A}, \mathbf{D}, \mathbf{B}\right)=L_{b a}\left(s, n_{A}, \mathbf{D},-\mathbf{B}\right), \\
& \mathrm{L}_{\alpha \beta}\left(s, n_{A}, \mathbf{D}, \mathbf{B}\right)=\mathrm{L}_{\beta \alpha}\left(s, n_{A}, \mathbf{D},-\mathbf{B}\right),
\end{aligned}
$$

which cannot be derived within a thermodynamic approach but require a statistical treatment since they are a consequence of the time reversibility of the microscopic dynamics [7]. The inequality (159) has to hold for each part, which implies that each quadratic form has to be positive definite.

Thus, the chemical reaction rate densities $\omega_{a}$ are related to the chemical affinities $\mathcal{A}_{b}$ through scalar linear phenomenological relations, i.e.,

$$
\omega_{a}=\sum_{b} L_{a b} \mathcal{A}_{b},
$$

where the Onsager matrix has to satisfy,

$$
\frac{1}{T}\left\{L_{a b}\right\} \geqslant 0,
$$

in order for the scalar quadratic form in the relation (159) to be positive definite. The scalar linear phenomenological relations (161) account for the dissipation due to the chemistry.

Similarly, the vectorial current densities $\mathbf{j}_{\alpha}$ are related to the vectorial forces $\mathbf{F}_{\beta}$ through vectorial linear phenomenological relations, i.e.,

$$
\mathbf{j}_{\alpha}=\sum_{\beta} \mathrm{L}_{\alpha \beta} \cdot \mathbf{F}_{\beta},
$$

where the Onsager matrix has to satisfy,

$$
\frac{1}{T}\left\{\mathrm{~L}_{\alpha \beta}\right\} \geqslant 0,
$$

in order for the vectorial quadratic form in the relation (159) to be positive definite. The vectorial linear phenomenological relations (163) are expressed explicitly in terms of the currents densities $\mathbf{j}_{s}$ and $\mathbf{j}_{A}$ and forces $\mathbf{F}_{S}$ and $\mathbf{F}_{A}$ as,

$$
\left\{\begin{array}{l}
\mathbf{j}_{s}=\mathrm{L}_{s s} \cdot(-\nabla T)+\sum_{B} \mathrm{~L}_{s B} \cdot \mathbf{F}_{B} \\
\mathbf{j}_{A}=\mathrm{L}_{A s} \cdot(-\nabla T)+\sum_{B} \mathrm{~L}_{A B} \cdot \mathbf{F}_{B}
\end{array}\right.
$$

where the Onsager matrix (164) is positive definite, i.e.,

$$
\frac{1}{T}\left(\begin{array}{ll}
\mathrm{L}_{s s} & \mathrm{~L}_{s B} \\
\mathrm{~L}_{A s} & \mathrm{~L}_{A B}
\end{array}\right) \geqslant 0 .
$$

The vectorial linear phenomenological relations (165) account for the dissipation due to the transport.

It is worth emphasising that although the scalar (161) and the vectorial (165) linear phenomenological relations are structurally independent, they are coupled through the time evolution equations. The chemical affinities $\mathcal{A}_{a}$ defined in relation (145) couple the scalar (161) and vectorial (165) linear phenomenological relations. This coupling has interesting applications for spintronics, some of which were investigated in [9].

In the particular case of a continuous medium made of a single chemical substance $A$, the scalar linear relations (161) vanish, the vectorial linear relations (165) become,

$$
\left\{\begin{array}{l}
\mathbf{j}_{S}=\mathrm{L}_{S S} \cdot(-\nabla T)+\mathrm{L}_{s A} \cdot \mathbf{F}_{A} \\
\mathbf{j}_{A}=\mathrm{L}_{A s} \cdot(-\nabla T)+\mathrm{L}_{A A} \cdot \mathbf{F}_{A}
\end{array}\right.
$$




\subsection{Physical applications}

\subsubsection{Navier-Stokes and Euler equations}

We consider a single chemical substance $A$, consisting of electrically neutral elementary units, i.e., $q_{A}=0$, that have no electric dipole, i.e., $\mathbf{p}_{A}=\mathbf{0}$, or magnetic moment, i.e., $\mathbf{m}_{A}=\mathbf{0}$, and that are homogeneously diluted, i.e., $\nabla \mu_{A}=\mathbf{0}$, in an electrically neutral and non-reactive viscous fluid at a uniform temperature, i.e., $\nabla T=\mathbf{0}$.

In the case where the elementary constituents have a uniform intrinsic angular velocity, i.e., $\nabla \omega_{A}=0$, using the relation (158), the linear phenomenological relation (167) reduces to,

$$
n_{A} m_{A} \mathbf{v}_{A} \nabla \mathbf{v}_{A}=-\eta_{A} \cdot\left(G_{t} \mathbf{j}_{A}\right),
$$

where

$$
\eta_{A} \equiv\left(G_{t} \mathrm{~L}_{A A}\right)^{-1}
$$

is the viscosity tensor, and $G_{t}$ is a geometric factor of unit length. The Eq. (168) is a tensorial generalisation of Stokes' law [10]. The symmetric viscosity tensor $\eta_{A}$ can be split irreducibly into a bulk viscosity scalar $\eta_{A}$ and a shear viscosity trace-free tensor $\tilde{\boldsymbol{\eta}}_{A}$, i.e.,

$$
\eta_{A}=\eta_{A} \mathbb{1}+\tilde{\eta}_{A} .
$$

The bulk viscosity scalar $\eta_{A}$ describes viscous friction where the friction force $m_{A} \mathbf{v}_{A} \nabla \mathbf{v}_{A}$ is collinear to the matter flow $\mathbf{j}_{A}$. The shear viscosity trace-free tensor $\tilde{\boldsymbol{\eta}}_{A}$ describes viscous friction where the friction force $m_{A} \mathbf{v}_{A} \boldsymbol{\nabla} \mathbf{v}_{A}$ is orthogonal to the matter flow $\mathbf{j}_{A}$.

In order to obtain the Navier-Stokes equation for an isotropic friction, i.e., $\tilde{\eta}_{A}=0$, the diffusive term in the time evolution equation (120) for the matter momentum has to be expressed in terms of the viscosity scalar $\eta_{A}$. Using the linear phenomenological relation (168), the diffusive term is recast as,

$$
-m_{A}\left(\mathbf{j}_{A} \cdot \boldsymbol{\nabla}\right) \mathbf{v}_{A}=-\left(\frac{n_{A} m_{A}^{2}}{G_{t} \eta_{A}} \mathbf{v}_{A} \boldsymbol{\nabla} \mathbf{v}_{A}\right) \cdot \boldsymbol{\nabla} \mathbf{v}_{A} .
$$

Near the equilibrium, the gradients of the fields, i.e., $\nabla \mathbf{v}_{A}, \nabla n_{A}, \nabla m_{A}$ and $\nabla \eta_{A}$ are sufficiently small. Thus, to first order in the gradient of the fields, the diffusive term (171) is recast as,

$$
-m_{A}\left(\mathbf{j}_{A} \cdot \nabla\right) \mathbf{v}_{A}=-\left(\nabla \hat{\eta}_{A}\right) \cdot \nabla \mathbf{v}_{A},
$$

where the viscosity scalar $\hat{\eta}_{A}$ is defined as,

$$
\hat{\eta}_{A}=\frac{1}{2} \frac{n_{A} m_{A}^{2}}{G_{t} \eta_{A}} \mathbf{v}_{A}^{2}
$$

The diffusive term (172) satisfies the vectorial identity,

$$
-\left(\nabla \hat{\eta}_{A}\right) \cdot \nabla \mathbf{v}_{A}=-\nabla \cdot\left(\hat{\eta}_{A} \nabla \mathbf{v}_{A}\right)+\hat{\eta}_{A} \nabla^{2} \mathbf{v}_{A},
$$

where the first term on the RHS corresponds to a surface contribution after integration over the volume of the continuum. Thus, it can be neglected in the bulk of the continuum, which implies that the diffusive term (172) reduces to,

$$
-m_{A}\left(\mathbf{j}_{A} \cdot \nabla\right) \mathbf{v}_{A}=\hat{\eta}_{A} \nabla^{2} \mathbf{v}_{A} .
$$

Substituting the expression (175) into the time evolution equation (120) for the matter momentum yields the Navier-Stokes equation, i.e.

$$
n_{A} m_{A} \dot{\mathbf{v}}_{A}=\mathbf{f}_{A}^{\text {ext }}+\mathbf{f}_{A}^{\text {em }}-\nabla P_{A}+\hat{\eta}_{A} \nabla^{2} \mathbf{v}_{A} .
$$

It is important to note that the Navier-Stokes equation (176) only applies for an isotropic fluid in the absence of a temperature gradient or an electrochemical gradient. Note that a generalised Navier-Stokes equation that accounts for the presence of thermodynamical field gradients can be deduced by applying our formalism. 
In the case where the elementary constituents have a uniform drift velocity, i.e., $\nabla \mathbf{v}_{A}=0$, using the relation (158), the linear phenomenological relation (167) reduces to,

$$
n_{A} I_{A} \omega_{A} \nabla \omega_{A}=-\gamma_{A} \cdot\left(G_{r} \mathbf{j}_{A}\right)
$$

where

$$
\gamma_{A} \equiv\left(G_{r} L_{A A}\right)^{-1}
$$

is the rotational viscosity tensor of the liquid solution, and $G_{r}$ is a geometric factor of unit length. The symmetric rotational viscosity tensor $\boldsymbol{\gamma}_{A}$ can be split irreducibly into a rotational viscosity scalar $\gamma_{A}$ and a rotational viscosity trace-free tensor $\tilde{\gamma}_{A}$, i.e.,

$$
\boldsymbol{\gamma}_{A}=\gamma_{A} \mathbb{1}+\tilde{\gamma}_{A} .
$$

The rotational viscosity scalar $\gamma_{A}$ describes viscous friction where the rotational friction force $I_{A} \omega_{A} \nabla \omega_{A}$ is collinear to the matter flow $\mathbf{j}_{A}$. The rotational viscosity trace-free tensor $\tilde{\gamma}_{A}$ describes viscous friction where the rotational friction force $I_{A} \omega_{A} \nabla \omega_{A}$ is orthogonal to the matter flow $\mathbf{j}_{A}$.

The expressions (169) and (178) for the tensorial viscosities are related through the geometric relation

$$
G_{t} \eta_{A}=G_{r} \boldsymbol{\gamma}_{A}
$$

which is a tensorial generalisation of Stoke's scalar relation that Debye [11] used in the particular case of a sphere.

In order to obtain the Euler equation for an isotropic friction, i.e., $\tilde{\gamma}_{A}=0$, the diffusive term in the time evolution equation (134) for the matter intrinsic angular momentum has to be expressed in terms of the rotational viscosity scalar $\gamma_{A}$. Using the linear phenomenological relation (177), the diffusive term is recast as,

$$
-I_{A}\left(\mathbf{j}_{A} \cdot \nabla\right) \omega_{A}=-\left(\frac{n_{A} m_{A}^{2}}{G_{r} \gamma_{A}} \omega_{A} \nabla \omega_{A}\right) \cdot \nabla \omega_{A}
$$

Near the equilibrium, the gradients of the fields, i.e., $\nabla \omega_{A}, \nabla n_{A}, \nabla I_{A}$ and $\nabla \gamma_{A}$ are sufficiently small. Thus, to first order in the gradient of the fields, the diffusive term (181) is recast as,

$$
-I_{A}\left(\mathbf{j}_{A} \cdot \nabla\right) \omega_{A}=-\left(\nabla \hat{\gamma}_{A}\right) \cdot \nabla \omega_{A},
$$

where the viscosity scalar $\hat{\gamma}_{A}$ is defined as,

$$
\hat{\gamma}_{A}=\frac{1}{2} \frac{n_{A} I_{A}^{2}}{G_{r} \gamma_{A}} \omega_{A}^{2} .
$$

The diffusive term (182) satisfies the vectorial identity,

$$
-\left(\nabla \hat{\gamma}_{A}\right) \cdot \nabla \omega_{A}=-\nabla \cdot\left(\hat{\gamma}_{A} \nabla \omega_{A}\right)+\hat{\gamma}_{A} \nabla^{2} \omega_{A},
$$

where the first term on the RHS corresponds to a surface contribution after integration over the volume of the continuum. Thus, it can be neglected in the bulk of the continuum, which implies that the diffusive term (182) reduces to,

$$
-I_{A}\left(\mathbf{j}_{A} \cdot \nabla\right) \omega_{A}=\hat{\gamma}_{A} \nabla^{2} \omega_{A}
$$

Substituting the expression (185) into the time evolution equation (134) for the matter momentum yields the Euler equation, i.e.,

$$
n_{A} I_{A} \dot{\boldsymbol{\omega}}_{A}=\boldsymbol{\tau}_{A}^{\mathrm{ext}}+\boldsymbol{\tau}_{A}^{\mathrm{em}}+\hat{\gamma}_{A} \nabla^{2} \boldsymbol{\omega}_{A}
$$

It is important to note that the Euler equation (186) only applies for an isotropic fluid in the absence of a temperature gradient or an electrochemical gradient. Note that a generalised Euler equation that accounts for the presence of thermodynamical field gradients can be deduced by applying our formalism. 


\subsubsection{Heat transport}

For a system consisting of a single chemical substance $A$ at rest in the local material frame, i.e., $\mathbf{j}_{A}=\mathbf{0}$, in the absence of viscous friction, i.e., $\nabla \mathbf{v}_{A}=\mathbf{0}$ and $\boldsymbol{\nabla} \boldsymbol{\omega}_{A}=\mathbf{0}$, the vectorial linear relations (165) account for heat transport, i.e.,

$$
\mathbf{j}_{Q}\left(s, n_{A}, \mathbf{D}, \mathbf{B}\right)=-\kappa\left(s, n_{A}, \mathbf{D}, \mathbf{B}\right) \cdot \nabla T\left(s, n_{A}, \mathbf{D}, \mathbf{B}\right),
$$

where $\mathbf{j}_{Q}=T \mathbf{j}_{s}$ is the heat current density, and $\boldsymbol{\kappa}\left(s, n_{A}, \mathbf{D}, \mathbf{B}\right)$ is the heat conductivity rank-2 tensor. The symmetric trace part of the heat conductivity tensor describes Fourier's law, and the antisymmetric part describes the Righi-Leduc effect [12]. Using the expression (139) for the temperature in terms of the matter and electromagnetic fields, the heat transport equation (187) is recast as,

$$
\begin{aligned}
& \mathbf{j}_{Q}\left(s, n_{A}, \mathbf{D}, \mathbf{B}\right)=-\kappa\left(s, n_{A}, \mathbf{D}, \mathbf{B}\right) \cdot\left(\nabla T\left(s, n_{A}, \mathbf{0}, \mathbf{0}\right)\right. \\
& \left.-\varepsilon_{0} \nabla\left(\frac{1}{2} \frac{\partial \chi_{e}^{A}}{\partial s} \cdot(\mathbf{E} \odot \mathbf{E})+\frac{1}{2} \frac{\partial \chi_{m}^{A}}{\partial s} \cdot(c \mathbf{B} \odot c \mathbf{B})+\frac{\partial \chi_{\alpha}^{A}}{\partial s} \cdot(\mathbf{E} \otimes c \mathbf{B})\right)\right) .
\end{aligned}
$$

\subsubsection{Chemical transport}

For a system consisting of an electrically neutral chemical substances $A$, i.e., $q_{A}=0$, in a fluid with a uniform temperature, i.e., $\boldsymbol{\nabla} T=\mathbf{0}$, in the absence of viscous friction, i.e., $\nabla \mathbf{v}_{A}=\mathbf{0}$ and $\boldsymbol{\nabla} \boldsymbol{\omega}_{A}=\mathbf{0}$, the vectorial linear relations (165) account for chemical transport, i.e.,

$$
\mathbf{j}_{A}\left(s, n_{A}, \mathbf{D}, \mathbf{B}\right)=-\mathrm{F}_{A}\left(s, n_{A}, \mathbf{D}, \mathbf{B}\right) \cdot \nabla \mu_{A}\left(s, n_{A}, \mathbf{D}, \mathbf{B}\right),
$$

where $\mathrm{F}_{A}\left(s, n_{A}, \mathbf{D}, \mathbf{B}\right)$ is the chemical diffusion rank-2 tensor of the electrically neutral chemical substance $A$. The symmetric trace part of the chemical diffusion tensor describes Fick's law [12]. Using the expression (142) for the chemical potential in terms of the matter and electromagnetic fields, the chemical transport equation (189) is recast as,

$$
\begin{aligned}
& \mathbf{j}_{A}\left(s, n_{A}, \mathbf{D}, \mathbf{B}\right)=-\mathrm{F}_{A}\left(s, n_{A}, \mathbf{D}, \mathbf{B}\right) \cdot\left(\nabla \mu_{A}\left(s, n_{A}, \mathbf{0}, \mathbf{0}\right)\right. \\
& \left.-\varepsilon_{0} \nabla\left(\frac{1}{2} \frac{\partial \chi_{e}^{A}}{\partial n_{A}} \cdot(\mathbf{E} \odot \mathbf{E})+\frac{1}{2} \frac{\partial \chi_{m}^{A}}{\partial n_{A}} \cdot(c \mathbf{B} \odot c \mathbf{B})+\frac{\partial \chi_{\alpha}^{A}}{\partial n_{A}} \cdot(\mathbf{E} \otimes c \mathbf{B})\right)\right) .
\end{aligned}
$$

\subsubsection{Thermochemical transport}

For a system consisting of electrically neutral chemical substances $A$ and $B$, i.e., $q_{A}=q_{B}=0$, at rest in the local material frame, i.e., $\mathbf{j}_{A}=\mathbf{j}_{B}=\mathbf{0}$, in the absence of viscous friction, i.e., $\boldsymbol{\nabla} \mathbf{v}_{A}=\mathbf{0}$ and $\boldsymbol{\nabla} \boldsymbol{\omega}_{A}=\mathbf{0}$, the vectorial linear relations (165) account for thermochemical transport, i.e.,

$$
\mathbf{j}_{Q}\left(s, n_{A}, \mathbf{D}, \mathbf{B}\right)=-\mathrm{D}_{A}\left(s, n_{A}, \mathbf{D}, \mathbf{B}\right) \cdot \nabla \mu_{A}\left(s, n_{A}, \mathbf{D}, \mathbf{B}\right),
$$

where $\mathrm{D}_{A}\left(s, n_{A}, \mathbf{D}, \mathbf{B}\right)$ is the thermochemical diffusion rank-2 tensor of the electrically neutral chemical substance $A$. The symmetric trace part of the thermochemical diffusion tensor describes the Dufour effect [12]. Using the expression (142) for the chemical potential in terms of the matter and electromagnetic fields, the thermochemical transport equation (191) is recast as,

$$
\begin{aligned}
& \mathbf{j}_{Q}\left(s, n_{A}, \mathbf{D}, \mathbf{B}\right)=-\mathrm{D}_{A}\left(s, n_{A}, \mathbf{D}, \mathbf{B}\right) \cdot\left(\nabla \mu_{A}\left(s, n_{A}, \mathbf{0}, \mathbf{0}\right)\right. \\
& \left.-\varepsilon_{0} \nabla\left(\frac{1}{2} \frac{\partial \chi_{e}^{A}}{\partial n_{A}} \cdot(\mathbf{E} \odot \mathbf{E})+\frac{1}{2} \frac{\partial \chi_{m}^{A}}{\partial n_{A}} \cdot(c \mathbf{B} \odot c \mathbf{B})+\frac{\partial \chi_{\alpha}^{A}}{\partial n_{A}} \cdot(\mathbf{E} \otimes c \mathbf{B})\right)\right) .
\end{aligned}
$$

At equilibrium, the thermochemical effect between two substances $A$ and $B$ is given by, i.e.,

$$
\nabla(\boldsymbol{\Delta} \mu)\left(s, n_{A}, n_{B}, \mathbf{D}, \mathbf{B}\right)=-\mathrm{S}_{A B}\left(s, n_{A}, n_{B}, \mathbf{D}, \mathbf{B}\right) \cdot \nabla T\left(s, n_{A}, n_{B}, \mathbf{D}, \mathbf{B}\right),
$$

where $\boldsymbol{\Delta} \mu\left(s, n_{A}, n_{B}, \mathbf{D}, \mathbf{B}\right)=\frac{\mu_{A}-\mu_{B}}{2}$ is the difference of chemical potential between the substances $A$ and $B$, and $\mathrm{S}_{A B}\left(s, n_{A}, n_{B}, \mathbf{D}, \mathbf{B}\right)$ is the equilibrium thermochemical rank-2 tensor of the electrically neutral chemical substances $A$ and $B$. The symmetric trace part of the equilibrium thermochemical tensor describes the Soret 
effect [12]. Using the expression (139) for the temperature in terms of the matter and electromagnetic fields, the thermochemical effect (193) is recast as,

$$
\begin{aligned}
& \nabla(\boldsymbol{\Delta} \mu)\left(s, n_{A}, n_{B}, \mathbf{D}, \mathbf{B}\right)=-\mathrm{S}_{A B}\left(s, n_{A}, n_{B}, \mathbf{D}, \mathbf{B}\right) \cdot\left(\nabla T\left(s, n_{A}, n_{B}, \mathbf{0}, \mathbf{0}\right)\right. \\
& \left.\quad-\sum_{i=A, B} \varepsilon_{0} \nabla\left(\frac{1}{2} \frac{\partial \chi_{e}^{i}}{\partial s} \cdot(\mathbf{E} \odot \mathbf{E})+\frac{1}{2} \frac{\partial \chi_{m}^{i}}{\partial s} \cdot(c \mathbf{B} \odot c \mathbf{B})+\frac{\partial \chi_{\alpha}^{i}}{\partial s} \cdot(\mathbf{E} \otimes c \mathbf{B})\right)\right) .
\end{aligned}
$$

\subsubsection{Thermoelectric transport}

For a system with a single homogeneous chemical substance $A$, i.e., $\nabla \mu_{A}=\mathbf{0}$, in the absence of chemical current densities, i.e., $\mathbf{j}_{A}=\mathbf{0}$, and of viscous friction, i.e., $\nabla \mathbf{v}_{A}=\mathbf{0}$ and $\nabla \boldsymbol{\omega}_{A}=\mathbf{0}$, the vectorial linear relations (165) account for thermoelectric transport, i.e.,

$$
\mathbf{E}\left(s, n_{A}, \mathbf{D}, \mathbf{B}\right)=\boldsymbol{\epsilon}\left(s, n_{A}, \mathbf{D}, \mathbf{B}\right) \cdot \nabla T\left(s, n_{A}, \mathbf{D}, \mathbf{B}\right),
$$

where $\boldsymbol{\epsilon}\left(s, n_{A}, \mathbf{D}, \mathbf{B}\right)$ is the thermoelectric conductivity rank-2 tensor. The symmetric trace part of the thermoelectric conductivity tensor describes the Seebeck effect, and the antisymmetric part describes the Nernst effect [12]. Using the expression (139) for the temperature in terms of the matter and electromagnetic fields, the thermoelectric transport equation (195) is recast as,

$$
\begin{aligned}
& \mathbf{E}\left(s, n_{A}, \mathbf{D}, \mathbf{B}\right)=\boldsymbol{\epsilon}\left(s, n_{A}, \mathbf{D}, \mathbf{B}\right) \cdot\left(\nabla T\left(s, n_{A}, \mathbf{0}, \mathbf{0}\right)\right. \\
& \left.-\varepsilon_{0} \nabla\left(\frac{1}{2} \frac{\partial \chi_{e}^{A}}{\partial s} \cdot(\mathbf{E} \odot \mathbf{E})+\frac{1}{2} \frac{\partial \chi_{m}^{A}}{\partial s} \cdot(c \mathbf{B} \odot c \mathbf{B})+\frac{\partial \chi_{\alpha}^{A}}{\partial s} \cdot(\mathbf{E} \otimes c \mathbf{B})\right)\right) .
\end{aligned}
$$

\section{Conclusion}

We determined the local thermodynamics of a classical continuous medium consisting of electrically charged reactive substances with spontaneous electric dipoles and magnetic moments in the presence of electromagnetic fields. We obtained expressions describing the local thermostatic equilibrium as well as the reversible and irreversible local thermodynamic evolution.

In order to achieve this, we made use of the continuity equations for the density fields, which were expressed in terms of tensorial current densities. Expressing the extensive matter state fields in terms of their chemical constituents, we obtained explicit expressions for the stress and the intrinsic angular stress tensors. We invoked the laws of thermodynamics expressed in a local frame and combined them with the laws of mechanics of continuous media. We also took into account Maxwell's equations for the electromagnetic fields and assumed linear constitutive relations with a magnetoelectric coupling.

The local equilibrium enabled us to obtain explicit expressions for the temperature and the chemical potential in terms of the electromagnetic fields. Thus, the transport equations, which are derived from the irreversible evolution, contain electromagnetic terms normally not included in a standard thermodynamic phenomenology.

Acknowledgments The authors would like to thank François A. Reuse for insightful discussions and thoughtful advise.

\section{References}

1. Stückelberg von Breidenbach, E.C.G., Scheurer, P.B.: Thermocinétique Phénoménologique Galiléenne. Birkhauser, Basel (1974)

2. Le Bellac, M., Lévy-Leblond, J.: Galilean electromagnetism. Il Nuovo Cimento B (1971-1996). 14, 217-218 (1973)

3. Muschik, W.: Objectivity and frame indifference of acceleration-sensitive materials. J. Theor. Appl. Mech. 50, 807 (2012)

4. Stratton, J.A.: Electromagnetic Theory, 1st edn. Wiley, New York (1941)

5. Lomholt, M.A., Miao, L.: Descriptions of membrane mechanics from microscopic and effective two-dimensional perspectives. Math. Gen. 39, 10323 (2006)

6. Santarelli, V.: Intrinsic angular momentum of light. Am. J. Phys. 47, 421 (1979)

7. Onsager, L.: Reciprocal Relations in Irreversible Processes. I. Phys. Rev. 37, 405 (1931)

8. Onsager, L.: Reciprocal Relations in Irreversible Processes. II. Phys. Rev. 38, 2265 (1931) 
9. Ansermet, J.P.: Thermodynamic description of spin mixing in spin-dependent transport. Magn. IEEE 44(3), 329 (2008)

10. Stokes, G.G.: On the effect of the internal friction on the motion of pendulums. Camb. Philos. Trans. 9, 8 (1851)

11. Debye, P.: Polar Molecules. Dover, New York (1929)

12. Callen, H.B.: Thermodynamics and an Introduction to Thermostatistics, 2nd edn. Wiley, New York (1960) 